\title{
PANSIONS AND THE THEORY OF FOURIER TRANSFORMS ${ }^{1}$
}

\author{
BY \\ JACOB KOREVAAR \\ INTRODUCTION
}

1. The classical Fourier transformation. The Fourier transform $g=T f$ of $f$ defined by

$$
g(x)=(T f)(x)=(2 \pi)^{-1 / 2} \int_{-\infty}^{\infty} f(u) e^{-i x u} d u
$$

will certainly exist if $f$ is of class $L_{1}$, that is, if $f$ is locally integrable and such that $|f|$ is integrable over $(-\infty, \infty)$. In that case $g$ will be continuous and bounded, but in general $g$ will not be of class $L_{1}$.

The function $h=\bar{T} f$ defined by

$$
h(x)=(\bar{T} f)(x)=(2 \pi)^{-1 / 2} \int_{-\infty}^{\infty} f(u) e^{i x u} d u
$$

is called the conjugate Fourier transform of $f$.

The famous inversion theorem for the Fourier transformation says that

$$
\text { if } g=T f \text { then } f=\bar{T} g \text {. }
$$

Unfortunately this basic result is true only under rather severe restrictions, for example if both $f$ and $T f$ are in $L_{1}$ [3, p. 51].

Other well-known rules of restricted validity are the following:

$$
\begin{aligned}
& T D f=\{i x\} T f, \quad T\{x\} f=i D T f, \\
& T E(D) f=\{E(i x)\} T f, \quad T\{E(x)\} f=E(i D) T f, \\
& T\left(f_{1} * f_{2}\right)=T f_{1} \cdot T f_{2} .
\end{aligned}
$$

Here $D$ stands for differentiation, $E(x)=\sum a_{n} x^{n}$ denotes an entire function, and $f_{1} * f_{2}$ stands for the convolution

$$
\left(f_{1} * f_{2}\right)(x)=(2 \pi)^{-1 / 2} \int_{-\infty}^{\infty} f_{1}(x-u) f_{2}(u) d u .
$$

The restrictions imposed by the classical theory make it hard to understand how Fourier transforms can be such a successful tool in physics and

Received by the editors June 19, 1957.

(1) This paper represents work which was supported by the United States Office of Naval Research. 
applied mathematics even when used indiscriminately. Thus one early came to the conclusion that more general theories of Fourier transforms must be possible, in which the above theorems are valid under more general conditions. Plancherel developed his theory for the class $L_{2}$ which shows that $L_{2}$ is closed under Fourier transformation (compare [21, p. 46]). Bochner defined his $k$-transform for all functions of polynomial growth [3, p. 110]. Schwartz imbedded the latter functions in his class of tempered distributions, which is closed under Fourier transformation [16, II, p. 79]. A theory of generalized functions and their Fourier transforms of comparable scope, but more along the lines of classical analysis, was developed by the present author $[11 ; 12]$. Using the Schwartz-Sobolev approach of distributions the theory of Fourier transforms was extended further by Gel'fand and Šilov [8] and by Ehrenpreis [7].

2. The present method of generalization. In this paper we develop a very general theory of Fourier transforms based upon a different set of ideas. The class of objects for which a Fourier transform will be defined is the class of formal series of normalized Hermite functions

$$
\phi=\sum_{k=0}^{\infty} c_{k} v_{k} .
$$

Some but not all of these objects are Hermite expansions of functions, and we therefore call the objects pansions. If $\phi$ is the Hermite expansion of a function $f$ of a suitable class we identify $\phi$ with $f$. Various operations on pansions will be defined, among them the Fourier transformation $[T]$ :

$$
[T]_{\phi}=\left(\sum_{k=0}^{\infty} c_{k} T v_{k}=\right) \sum_{k=0}^{\infty}(-i)^{k} c_{k} v_{k} .
$$

There is no natural definition of convergence for pansions as there is for distributions. As a matter of fact no concept of convergence is introduced in this paper and accordingly our theory is more or less algebraic in nature.

The following considerations led to the selection of series of Hermite functions. Let $u_{0}, u_{1}, \cdots$ be any orthonormal set of functions on $(-\infty, \infty)$ which is complete with respect to a preferably large class of functions $C$. We will take $C$ such that it contains the difference of any two of its elements. A function $f$ of $C$ is uniquely determined by its expansion

$$
f \sim \sum d_{k} u_{k} \quad \text { where } \quad d_{k}=\left(f, u_{k}\right)=\int_{-\infty}^{\infty} f(x) u_{k}(x) d x .
$$

For if $f$ and $g$ of $C$ have the same expansion then $f-g \in C$ is orthogonal to all $u_{k}$ and hence by the completeness of the $u_{k}$ we conclude that $f-g=0$. There is no need to talk about convergence of the expansion.

To make a definition 


$$
[T] \sum c_{k} u_{k}=\sum c_{k} T u_{k}
$$

meaningful the ordinary Fourier transforms $T u_{k}$ should exist and be simple linear combinations of the $u$ 's. The simplest theory will be obtained if $T u_{k}$ is just a multiple of $u_{k}$. We are thus led to search for a boundary value problem

$$
H y=l y,
$$

such that the differential operator $H$ commutes with Fourier transformation, and such that the Fourier transform of a function satisfying the boundary condition (B) again satisfies (B). For if $\lambda, u$ is a characteristic pair of such a boundary value problem $(H u=\lambda u)$, then so is $\lambda, T u$. Hence if the boundary value problem has essentially only one eigenfunction $u$ to each eigenvalue $\lambda$ we may conclude that $T u=c u, c$ a constant.

The simplest differential operator which commutes with Fourier transformation is $H=\left\{x^{2}\right\}-D^{2}$, and we are thus led to the boundary value problem of the linear harmonic oscillator

$$
H y \equiv\left(x^{2}-D^{2}\right) y=l y, \quad 0<\int_{-\infty}^{\infty}|y(x)|^{2} d x<\infty .
$$

Accordingly our set of $u_{k}$ 's will be the set of normalized Hermite functions

$$
v_{k}(x)=(k !)^{-1 / 2} 2^{-k / 2} \pi^{-1 / 4}(x-D)^{k} e^{-x^{2} / 2}, \quad k=0,1, \ldots
$$

for which

$$
T v_{k}=(-i)^{k} v_{k}, \quad \bar{T} v_{k}=i^{k} v_{k} .
$$

3. Contents of the paper. In I we treat the linear harmonic oscillator using the method of factorization or method of adjoint operators. This method brings out some of the properties of $H=\left\{x^{2}\right\}-D^{2}$ which are basic for the paper, and it provides the convenient definition (2.1) of the Hermite functions.

In II it is shown that the Hermite functions are complete with respect to the class $A$ of functions $f$ for which there exists a number $a<1 / 2$ such that $f(x) \exp \left(-a x^{2}\right)$ is of class $L_{1}$. We study Hermite expansions and expansion coefficients of functions of class $A$. The expansions of $\{x\} f, D f, H f$ and $T f$ are expressed in terms of the expansion coefficients $d_{k}$ of $f$. It is important to notice that every expansion coefficient of these transforms of $f$ involves only a finite number of $d_{k}$ 's.

As an application IV contains a simple proof of the Fourier inversion theorem in the case where $f$ and $T f$ are of class $L_{1}$.

In $\mathrm{V}$ we define a pansion $\phi$ as a formal series $\sum c_{k} v_{k}$ where the $v_{k}$ are the normalized Hermite functions (2.1). If $\phi$ is the expansion of a function $f$ of class $A$ or a suitable larger class we identify $\phi$ with $f$. The global derivative 
$[D] \phi$, the global product $[x] \phi$ and $[H] \phi$ are defined for all pansions $\phi$ in accordance with the rules valid for the expansions of the corresponding transforms of functions $f$ of $A$. We also define $[E(D)] \phi,[E(x)] \phi$ and $[E(H)] \phi$ where $E$ is an element of a class of entire functions appropriate to $\phi$. The operator $\left[e^{a D}\right]$ plays the role of a translation operator: for every function $f$ of class $A$ and for real $a$ one has $\left[e^{a D}\right] f=\{f(x+a)\}$ (Taylor's formula!). Global multiplication of two pansions is considered, as well as convolution. (For a more detailed local study of multiplication and convolution, important for certain applications, compare the author's paper [12].)

VI deals with Fourier transforms of pansions. If $\phi=\sum c_{k} v_{k}$ we define

$$
\begin{aligned}
& {[T]_{\phi}=\left(\sum c_{k} T v_{k}=\right) \sum(-i)^{k} c_{k} v_{k},} \\
& {[\bar{T}]_{\phi}=\left(\sum c_{k} \bar{T} v_{k}=\right) \sum i^{k} c_{k} v_{k} .}
\end{aligned}
$$

If $\phi$ is a function $f$ of class $L_{1}$ then $[T] \phi=T f$. For pansions the Fourier inversion formula will hold without restriction, and likewise such rules as

$$
[T][D]_{\phi}=i[x][T] \phi, \quad[T][x]_{\phi}=i[D][T]_{\phi} .
$$

Other rules will also be valid under much weaker hypotheses than one has in the classical theory.

As an application VII contains a very simple derivation of Plancherel's theory of Fourier transforms for $L_{2}$. This derivation shows some similarity with Wiener's treatment based on Hermite expansions [21, p. 46], but it is considerably shorter.

VIII deals with Fourier transform theory for pansions of polynomial growth. Using global properties of the solutions of the differential equation $H y=f$ it was shown in III that every function of at most polynomial growth on $(-\infty, \infty)$ can be represented in the form $H^{n} f_{0}$ where $f_{0}$ is a sufficiently differentiable function of class $L_{2}$. Since $[T]$ and $[H]$ commute $[T] H^{n} f_{0}$ $=[H]^{n} g_{0}$ where $g_{0}=[T] f_{0}$ is also of class $L_{2}$. Defining pansions of polynomial growth as pansions of the form

$$
\phi=[H]{ }^{n} f_{1} \quad n \geqq 0, f_{1} \in L_{2},
$$

we thus obtain a class of pansions closed under Fourier transformation which contains all functions of at most polynomial growth. The class is also closed under the operations of global differentiation $[D]$, global multiplication by $[x]$ and translation $\left[e^{a D}\right], a$ real. It is shown that $a$ pansion is of polynomial growth if and only if it is a finite order global derivative of a function of at most polynomial growth. Hence the pansions of polynomial growth differ from Schwartz's tempered distributions only in that the latter have a natural definition of convergence associated with them [16, II, p. 95]. It is finally noted that for a function $f$ of polynomial growth $[T] f$ is essentially equal to the global $k$ th derivative of Bochner's " $k$-Transformierte" of $f[3$, p. 110].

In IX we take up Fourier transform theory for pansions of exponential 
growth. In III a rather precise study was made of the global properties of the solutions of the differential equation $(H+p) y=f$. The results showed that every function $f$ of at most exponential growth on $(-\infty, \infty)$ (of type $m$ ) can be represented in the form $E(H) f_{0}$. Here $f_{0}$ is an infinitely differentiable function of class $L_{2}, E\left(z^{2}\right)=c z^{2} \Pi\left(1+z^{2} / p_{j}\right)$ is a special entire function of exponential type (of type $m$ ), and the representation holds in the sense that

$$
f=H f^{*}, \quad f^{*}=\lim _{n \rightarrow \infty} c \prod_{j \leqq n}\left(1+H / p_{j}\right) f_{0} .
$$

Since $[T]$ and $[E(H)]$ commute $[T] E(H) f_{0}=[E(H)] g_{0}$ where $g_{0}=[T] f_{0}$ is also of class $L_{2}$. Defining pansions of exponential growth as pansions of the form

$$
\phi=[E(H)] f_{1}, \quad f_{1} \in L_{2}
$$

where $E\left(z^{2}\right)$ is any even entire function of exponential type, one thus obtains a class of pansions closed under Fourier transformation which contains all functions of at most exponential growth. The class is also closed under the operations $[E(D)]$ and $[E(x)]$ where $E$ is any entire function of exponential type. It is finally shown that a pansion is of exponential growth if and only if it can be written as an infinite order global derivative of exponential type of a function of at most exponential growth. The proof depends on explicit solution of the equation

$$
\phi=[\cos a D] f, \quad a>0 .
$$

If $\phi=[E(H)] f_{1}, f_{1} \in L_{2}, E\left(z^{2}\right)$ of exponential type, then a particular solution of the above equation is given by

$$
\begin{aligned}
f & =\frac{1}{\prod_{j \leq s}\left(1-D^{2} / r_{j}{ }^{2}\right)} g, \quad r_{j}=(j-1 / 2) \pi / a, \\
g(x) & =\frac{2}{1} \cdots \frac{2 s}{2 s-1} \frac{1}{2 a} \int_{-\infty}^{\infty} f_{1}(u) E(H) \frac{1}{[\cosh \{\pi(x-u) / 2 a\}]^{2 s+1}} d u,
\end{aligned}
$$

$H=u^{2}-d^{2} / d u^{2}$. For given $\phi$ it is necessary to take $a$ and $s$ sufficiently large to guarantee the existence of $g$ (and hence of $f$ ) as a function of at most exponential growth.

\section{THE LINEAR HARMONIC OSCILLATOR}

11. The boundary value problem. The linear harmonic oscillator in quantum mechanics poses the boundary value problem $[20$, p. 56]

$$
\begin{gathered}
H y \equiv\left(x^{2}-D^{2}\right) y=l y, \quad D=d / d x, \\
0<\int_{-\infty}^{\infty}|y(x)|^{2} d x<\infty .
\end{gathered}
$$


A function $f$ is said to be of class $I_{p}$ if it is so smooth that it can be written as a $p$ times iterated indefinite integral of an integrable function. By the usual definition $[4$, p. 42] a solution $f$ of the differential equation (11.1) must be of class $I_{2}$. (Since $D^{2} f=\left(x^{2}-l\right) f$ the solutions $f$ will actually be of class $I_{p}$ for every $p$.) Hence the second inequality of (11.2) is truly a boundary condition as $x \rightarrow \pm \infty$.

In the physical problem the expression $|y(x)|^{2}$ is a probability density: $\int_{a}^{b}|y(x)|^{2} d x$ represents the probability to find the particle on the interval $(a, b)$ when its energy is given by $l$. The values $\lambda$ of $l$ for which equations (11.1) and (11.2) possess a solution $v$ for $y$ represent the possible energy levels of the particle. Physically speaking one therefore expects that all eigenvalues $\lambda$ will be real and non-negative.

If $v$ is an eigenfunction belonging to the real or complex eigenvalue $\lambda$ we will call $\lambda, v$ a characteristic pair of the boundary value problem.

Let $L_{p}$ denote the class of all functions $f$ defined on $(-\infty, \infty)$ which are locally integrable and such that $|f|^{p}$ is integrable over $(-\infty, \infty)$. We will use the scalar product notation

$$
(f, g)=\int_{-\infty}^{\infty} f(x) \bar{g}(x) d x
$$

whenever the product $f \bar{g}$ is of class $L_{1}$, hence in particular if both $f$ and $g$ are in $L_{2}$.

12. Factorization of the operator $H$. The factorization method, also called the method of adjoint operators, was first used by Dirac [5, p. 137] for the problem of the linear oscillator. It was formulated as a method by Schrödinger [15] and subsequently developed by him, Infeld and other authors [10]. The present paper gives a rigorous version in the special case (11.1) $+(11.2)$.

Introducing the operators

$$
x+D=P, \quad x-D=N
$$

the operator $H=x^{2}-D^{2}$ may be "factored" as follows:

$$
H=P N-1=N P+1 .
$$

Thus the differential equation (11.1) may be written in each of the equivalent forms

$$
H y=l y, \quad P N y=(l+1) y, \quad N P y=(l-1) y .
$$

We note also that $P$ and $N$ are formally adjoint in the sense that $(P f) g$ $-f(N g)=D(f g)$.

Let $f(x)=f(x, l)$ be a solution of the differential equation (12.1). Then $f$ belongs to every class $I_{p}(11)$, hence $P f \in I_{2}$ and

$$
P N(P f)=P(N P f)=P\{(l-1) f\}=(l-2+1) P f,
$$


that is, $P f$ will be a solution of (12.1) with $l$ replaced by $l-2$. Similarly $N f$ will be a solution of (12.1) with $l$ replaced by $l+2$.

For the boundary value problem 11 we need the nonzero solutions of (12.1) which are of class $L_{2}$. We shall see in a moment that if $f$ is a solution of (12.1) of class $L_{2}$ then $P f$ and $N f$ are also in $L_{2}$. To prove this and further results we need the following theorem.

12.2. Theorem. (i) If $v$ is in $D_{2}=I_{2} \cap L_{2}$ and such that $H v \in L_{2}$ then Pv and $N v$ are in $D_{1}=I_{1} \cap L_{2}$.

(ii) $P$ and $N$ are adjoint operators in the sense that

$$
(P f, g)=(f, N g)
$$

for every $f$ and $g$ of $I_{1}$ for which $(f, g)$ and the above scalar products exist.

The proof of 12.2 will be postponed till 14 so as not to interrupt the present argument.

Let $\lambda, v$ be a characteristic pair of the boundary value problem 11 . Then $v$ and $H v=\lambda v$ are in $D_{2}$, hence by 12.2 (i) $P v=w$ is in $D_{1}$. By (12.1) $N w=N P v$ $=(\lambda-1) v$, hence $N w$ is in $L_{2}$. Application of 12.2 (ii) shows that

$$
(P v, P v)=(P v, w)=(v, N w)=(\bar{\lambda}-1)(v, v) .
$$

Similarly $N v=z$ is in $D_{1}$ while $P z=P N v=(\lambda+1) v$ is in $L_{2}$. Thus

$$
(N v, N v)=(z, N v)=(P z, v)=(\lambda+1)(v, v) .
$$

The preceding results imply the following basic theorem.

12.5. Theorem. Let $\lambda, v$ be any characteristic pair of the boundary value problem 11. Then $\lambda$ is real and $\geqq 1$ (12.3). If $\lambda=1$ then $P v=0$, while if $\lambda>1$ then $P v \neq 0$ and the pair $\lambda-2, P v$ is also a characteristic pair. The pair $\lambda+2$, $N v$ is always a characteristic pair (12.4).

13. The characteristic pairs. Let $\lambda$, $v$ be any characteristic pair of the boundary value problem 11 . Repeated application of 12.5 shows that either $\lambda=1$ and $P v=0$, or $\lambda-2=1$ and $P^{2} v=0$, or $\cdots$. That is, $\lambda$ must be of the form $2 k+1$ for some non-negative integer $k$, and

$$
P^{k+1} v=0 .
$$

For $k=0$ equation (13.1) has the solution $\exp \left(-x^{2} / 2\right)$ (unique up to a constant factor) which actually is an eigenfunction of $H$ belonging to the eigenvalue 1.

Repeated application of the last line of 12.5 , starting with the characteristic pair 1, exp $\left(-x^{2} / 2\right)$, now shows that each of the pairs

$$
2 k+1, N^{k} e^{-x^{2} / 2},
$$$$
k=0,1, \cdots
$$

is a characteristic pair of the boundary value problem. 
We will prove by induction that there is essentially only one eigenfunction belonging to the eigenvalue $2 k+1$. Let $v_{1}$ and $v_{2}$ be any two eigenfunctions belonging to $2 k+1(k \geqq 1)$, and suppose it has been shown already that there are no two linearly independent eigenfunctions to the eigenvalue $2 k-1$. In particular $P v_{1}$ and $P v_{2}$ will be linearly dependent, hence there are constants $c_{1}$ and $c_{2}$ not both zero such that

$$
P\left(c_{1} v_{1}+c_{2} v_{2}\right)=c_{1} P v_{1}+c_{2} P v_{2}=0 .
$$

It follows that $c_{1} v_{1}+c_{2} v_{2}=c \exp \left(-x^{2} / 2\right)$. Unless equal to zero $c_{1} v_{1}+c_{2} v_{2}$ is an eigenfunction belonging to $2 k+1 \geqq 3$ while $\exp \left(-x^{2} / 2\right)$ is an eigenfunction belonging to 1 . Thus $c=0$ and the result follows.

Hence the set (13.2) is a complete set of characteristic pairs of the problem.

Equation (12.4) enables us to obtain a normalized eigenfunction belonging to the eigenvalue $2 k+1$ in the form

$$
v_{k}(x)=\frac{1}{(2 k)^{1 / 2}}(x-D) v_{k-1}(x)=\frac{1}{2^{k / 2}(k !)^{1 / 2} \pi^{1 / 4}}(x-D)^{k} e^{-x^{2} / 2}
$$

(normalized Hermite function).

We finally show that the $v_{k}$ form an orthogonal set. If $m>k$ we have by 12.2 and (13.1)

$$
\left(v_{k}, v_{m}\right)=\left(v_{k}, a_{m} N^{m} e^{-x^{2} / 2}\right)=\left(P^{m} v_{k}, a_{m} e^{-x^{2} / 2}\right)=0 .
$$

14. Proof of 12.2. (i) Let $v$ be in $D_{2}=I_{2} \cap L_{2}$ and let $H v \in L_{2}$. Then the function

$$
\operatorname{Re} H v \cdot \bar{v}=x^{2}|v(x)|^{2}-\operatorname{Re}\left\{D^{2} v(x) \cdot \bar{v}(x)\right\}
$$

is integrable over $(-\infty, \infty)$, hence the expression

$$
\begin{aligned}
\int_{a}^{b} \operatorname{Re} H v \cdot \bar{v} d x= & \int_{a}^{b}\left\{x^{2}|v(x)|^{2}+|D v(x)|^{2}\right\} d x \\
& -[\operatorname{Re}\{D v(x) \cdot \bar{v}(x)\}]_{a}^{b}
\end{aligned}
$$

has a finite limit as $a \rightarrow-\infty, b \rightarrow \infty$.

We note that there must exist a sequence $b_{n} \rightarrow \infty$ along which the continuous function $w(x)=\operatorname{Re}\{D v(x) \cdot \bar{v}(x)\}$ approaches zero. For if this were false there would be numbers $\delta>0$ and $\beta$ such that $\pm w(x) \geqq \delta$ for all $x>\beta$. And this would contradict the fact that the anti-derivative $|v(x)|^{2} / 2$ of $w(x)$ is integrable over $(-\infty, \infty)$. One similarly shows that there exists a sequence $a_{m} \rightarrow-\infty$ along which $w(x) \rightarrow 0$.

We conclude that

$$
\lim _{m, n \rightarrow \infty} \int_{a_{m}}^{b_{n}}\left\{x^{2}|v(x)|^{2}+|D v(x)|^{2}\right\} d x
$$


exists and is finite. Since the integrand is non-negative this shows that it is integrable over $(-\infty, \infty)$, hence both $x v(x)$ and $D v(x)$ are in $L_{2}$. They obviously are in $I_{1}$.

(ii) Let $f$ and $g$ be in $I_{1}$ and such that $(f, g),(P f, g)$ and $(f, N g)$ exist. Since $(P f) \bar{g}-f(N \bar{g})=D(f \bar{g})$ and $f \bar{g} \in I_{1}$ one has for every finite $a$ and $b$

$$
\int_{a}^{b}(P f \cdot \bar{g}-f \cdot N \bar{g}) d x=[f(x) \bar{g}(x)]_{a}^{b} .
$$

The function in square brackets is absolutely integrable over $(-\infty, \infty)$. Hence there exist sequences $a_{m} \rightarrow-\infty, b_{n} \rightarrow \infty$ along which $f \bar{g}$ approaches zero. Thus since the scalar products $(P f, g)$ and $(f, N g)$ exist,

$$
\begin{aligned}
(P f, g) & =\lim _{m, n \rightarrow \infty} \int_{a_{m}}^{b_{n}} P f \cdot \bar{g} d x \\
& =\lim _{m, n \rightarrow \infty} \int_{a_{m}}^{b_{n}} f \cdot N \bar{g} d x=(f, N g) .
\end{aligned}
$$

14.1 REMARK. Using part (i) of the proof it is easy to show that $H$ is selfadjoint and positive definite on $D_{2}$. That is,

$$
(H f, g)=(f, H g)
$$

for all $f$ and $g$ in $D_{2}$ such that $H f$ and $H g$ are in $D_{2}$, and

$$
(H f, f)>0
$$

for all $f \neq 0$ in $D_{2}$ such that $H f$ is in $D_{2}$.

We will later use the sharper result that $(H f, g)=(f, H g)$ as soon as $f$ and $g$ are in $I_{2}$ and such that $(D f, g),(H f, g),(f, D g)$ and $(f, H g)$ exist.

\section{Hermite expansions}

21. The Hermite functions. (Compare [17, p. 101].) By $v_{k}$ we will always denote the normalized Hermite function (13.3)

$$
v_{k}(x)=\frac{1}{2^{k / 2}(k !)^{1 / 2} \pi^{1 / 4}}(x-D)^{k} e^{-x^{2} / 2}, \quad k=0,1, \cdots .
$$

It is easily seen that $v_{k}$ is even if $k$ is even and odd if $k$ is odd, and that $v_{k}(x)$ is equal to the product of $\exp \left(-x^{2} / 2\right)$ and a polynomial in $x$ of exact degree $k$. The latter remark will be used to prove the completeness theorem stated below.

21.2. The class $A$. A function $f$ is said to be of class $A$ if there is a real number $a<1 / 2$ such that $f(x) \cdot \exp \left(-a x^{2}\right)$ is integrable (and absolutely integrable) over $(-\infty, \infty)$.

21.3. Completeness theorem. The set of the Hermite functions (21.1) is 
complete with respect to the class $A$. That is, the equations

$$
\left(f, v_{k}\right)=\int_{-\infty}^{\infty} f(x) v_{k}(x) d x=0, \quad k=0,1, \cdots
$$

for a function $f \in A$ imply that $f=0(f(x)=0$ except perhaps on a set of measure zero).

In 21.3 the class $A$ may be replaced by a somewhat larger class. However, the simplest proof of the more general result is based on Fourier transform theory. At this stage we want to avoid using results about Fourier transforms and therefore we postpone the more general result till 42 .

Proof of 21.3. Let $\left(f, v_{k}\right)=0, f \in A$. Then

$$
\int_{-\infty}^{\infty} f(x) e^{-x^{2} / 2} x^{k} d x=0, \quad k=0,1, \cdots
$$

It follows that the function

$$
g(z)=\int_{-\infty}^{\infty} x f(x) e^{-z x^{2}} d x
$$

which is analytic for $\operatorname{Re} z>a$ for some $a<1 / 2$ vanishes at $z=1 / 2$ together with all its derivatives. Thus $g(z)=0$, that is, the Laplace transform

$$
\int_{0}^{\infty}\left\{f\left(y^{1 / 2}\right)-f\left(-y^{1 / 2}\right)\right\} e^{-z y} d y
$$

is equal to 0 for $z>a$.

Applying the uniqueness theorem for the Laplace transformation [6, p. 35] which is equivalent to a simple moment theorem one concludes that the odd part of $f$ is equal to 0 . Similarly the even part is 0 , hence $f=0$.

21.4. Corollary. The Hermite functions (21.1) form a complete orthonormal set in $L_{2}$.

For if $f \in L_{2}$ then $\left(1+x^{2}\right)^{-1 / 2} f(x) \in L_{1}$, hence if $f \in L_{2}$ is orthogonal to all $v_{k}$ then $f=0$. For the orthonormality see 13 .

22. Formulas involving the Hermite functions. In what follows $\{f(x)\}$ will denote the function whose value at $x$ is equal to $f(x)$. As before we will set

$$
\{x\}+D=P, \quad\{x\}-D=N, \quad\left\{x^{2}\right\}-D^{2}=H .
$$

By 12 and 13 we have the relations

$$
\begin{aligned}
& P v_{k}=(2 k)^{1 / 2} v_{k-1}, \\
& N v_{k}=(2 k+2)^{1 / 2} v_{k+1}, \\
& H v_{k}=(2 k+1) v_{k} .
\end{aligned}
$$


Thus

$$
\begin{aligned}
D v_{k} & =\left(\frac{1}{2} k\right)^{1 / 2} v_{k-1}-\left(\frac{1}{2} k+\frac{1}{2}\right)^{1 / 2} v_{k+1}, \\
\{x\} v_{k} & =\left(\frac{1}{2} k\right)^{1 / 2} v_{k-1}+\left(\frac{1}{2} k+\frac{1}{2}\right)^{1 / 2} v_{k+1} .
\end{aligned}
$$

By (22.2) and (21.1)

$$
\begin{aligned}
\int_{-\infty}^{\infty} v_{2 j}(x) d x & =\frac{\{(2 j) !\}^{1 / 2} \pi^{1 / 4}}{2^{j-1 / 2} j !} \\
v_{2 j}(0) & =\frac{(-1)^{j}\{(2 j) !\}^{1 / 2}}{2^{i j} !^{1 / 4}} .
\end{aligned}
$$

We finally compute the Fourier transform $T v_{k}$ of $v_{k}$. Since for functions $f \in I_{1} \cap L_{1}$ such that $\{x\} f$ and $D f$ are also in $L_{1}$

$$
T N f=-i N T f
$$

(compare 1) definition (21.1) shows that

$$
T v_{k}=T a_{k} N^{k}\left\{e^{-x^{2} / 2}\right\}=(-i)^{k} a_{k} N^{k} T\left\{e^{-x^{2} / 2}\right\} .
$$

It is easily verified that $T\left\{\exp \left(-x^{2} / 2\right)\right\}=\left\{\exp \left(-x^{2} / 2\right)\right\}$, hence

$$
T v_{k}=(-i)^{k} v_{k}, \quad \bar{T} v_{k}=i^{k} v_{k} .
$$

23. Hermite expansions of functions of class $A$. The Hermite expansion of a function $f \in A$ (21.2) is defined as the formal infinite series

$$
f \sim \sum_{k=0}^{\infty} c_{k} v_{k}
$$

where

$$
c_{k}=\left(f, v_{k}\right)=\int_{-\infty}^{\infty} f(x) v_{k}(x) d x .
$$

23.3. EXAMPLe. By (22.3) and since $v_{2 j+1}$ is odd,

$$
\{1\} \sim \sum_{j=0}^{\infty} \frac{\{(2 j) !\}^{1 / 2} \pi^{1 / 4}}{2^{j-1 / 2} j !} v_{2 j} .
$$

The Completeness Theorem 21.3 implies

23.4. Uniqueness theorem. If two functions of $A$ have the same Hermite expansion they are equal (their values are equal almost everywhere).

A stronger uniqueness theorem will be proved in 42 . 
Expansion of $\{x\} f$. Let $f$ be in $A, f \sim \sum c_{k} v_{k}$. Then $\{x\} f$ is in $A$. Formally, by $(22.2)$,

$$
\{x\} f \sim \sum c_{k}\{x\} v_{k} \sim \sum c_{k}\left\{\left(\frac{1}{2} k\right)^{1 / 2} v_{k-1}+\left(\frac{1}{2} k+\frac{1}{2}\right)^{1 / 2} v_{k+1}\right\},
$$

and by rearrangement (compare [20, p. 59])

$$
\{x\} f \sim \sum_{k=0}^{\infty}\left\{\left(\frac{1}{2} k+\frac{1}{2}\right)^{1 / 2} c_{k+1}+\left(\frac{1}{2} k\right)^{1 / 2} c_{k-1}\right\} v_{k} .
$$

Formula (23.5) is really correct. For by (22.2)

$$
\left(\{x\} f, v_{k}\right)=\left(f,\{x\} v_{k}\right)=\left(\frac{1}{2} k\right)^{1 / 2}\left(f, v_{k-1}\right)+\left(\frac{1}{2} k+\frac{1}{2}\right)^{1 / 2}\left(f, v_{k+1}\right) .
$$

Applying (23.5) twice one obtains

$$
\begin{aligned}
\left\{x^{2}\right\} f \sim \sum_{k=0}^{\infty} \frac{1}{2}\left\{(k+2)^{1 / 2}(k\right. & +1)^{1 / 2} c_{k+2} \\
& \left.+2\left(k+\frac{1}{2}\right) c_{k}+k^{1 / 2}(k-1)^{1 / 2} c_{k-2}\right\} v_{k} .
\end{aligned}
$$

Expansion of Ef. Let $E(x)=\sum a_{n} x^{n}$ be an entire function such that

$$
\sum\left|a_{n} x^{n}\right| \leqq C e^{a x^{2}}
$$

and let $f(x) \exp \left(-b x^{2}\right) \in L_{1}$ for some $b$ such that $a+b<1 / 2$. Then the sequence

$$
\sum_{n=0}^{p} a_{n} x^{n} f(x) v_{k}(x)
$$$$
p=0,1, \cdots
$$

is dominated by a function of $L_{1}$, hence by Lebesgue's convergence theorem $[18$, p. 345]

$$
\left(E f, v_{k}\right)=\left(\left\{\sum a_{n} x^{n}\right\} f, v_{k}\right)=\sum a_{n}\left(\{x\}^{n} f, v_{k}\right) .
$$

24. Expansion of $D f, T f, H f$.

Expansion of $D f$. Let $f$ be of class $I_{1}(11)$, and let the derivative $D f$ be in $A$. Then $f$ is in $A$ and bounded by a constant multiple of $\left\{e a x^{2}\right\}, a<1 / 2$. Hence by integration by parts and by $(22.2)$

$$
\left(D f, v_{k}\right)=-\left(f, D v_{k}\right)=-\left(\frac{1}{2} k\right)^{1 / 2}\left(f, v_{k-1}\right)+\left(\frac{1}{2} k+\frac{1}{2}\right)^{1 / 2}\left(f, v_{k+1}\right) .
$$

Thus if $f \sim \sum c_{k} v_{k}$ then

$$
D f \sim \sum_{k=0}^{\infty}\left\{\left(\frac{1}{2} k+\frac{1}{2}\right)^{1 / 2} c_{k+1}-\left(\frac{1}{2} k\right)^{1 / 2} c_{k-1}\right\} v_{k} .
$$


Expansion of $T f$. Let $f$ be in $L_{1}$. By Fubini's theorem $[14$, p. 77] we may invert the order of integration in the formula below to obtain the expansion coefficients of the Fourier transform $T f$ (see also (22.4)):

$$
\begin{aligned}
\left(T f, v_{k}\right) & =(2 \pi)^{-1 / 2} \int_{-\infty}^{\infty} v_{k}(x) d x \int_{-\infty}^{\infty} f(u) e^{-i x u} d u \\
& =\int_{-\infty}^{\infty} f(u) d u \cdot(2 \pi)^{-1 / 2} \int_{-\infty}^{\infty} v_{k}(x) e^{-i x u} d x=(-i)^{k}\left(f, v_{k}\right) .
\end{aligned}
$$

Hence if $f \sim \sum c_{k} v_{k}$ then

$$
T f \sim \sum_{k=0}^{\infty}(-i)^{k} c_{k} v_{k}
$$

Similarly

$$
\bar{T} f \sim \sum_{k=0}^{\infty} i^{k} c_{k} v_{k} .
$$

Expansion of $H f$. Let $f$ be in $I_{2}$ and such that $D^{2} f \in A$. Then $D f \in A, f \in A$, and if $f \sim \sum c_{k} v_{k}$ then

$$
H f \sim \sum_{k=0}^{\infty}(2 k+1) c_{k} v_{k}
$$

For by 14.1 and (22.1)

$$
\left(H f, v_{k}\right)=\left(f, H v_{k}\right)=(2 k+1)\left(f, v_{k}\right) .
$$

Expansion of $E(D) f$ and $E(H) f$. For sufficiently well-behaved functions $f$ and sufficiently restricted entire functions $E(x)=\sum a_{n} x^{n}$

$$
\begin{aligned}
\left(E(D) f, v_{k}\right) & =\left(\left(\sum a_{n} D^{n}\right) f, v_{k}\right)=\sum a_{n}\left(D^{n} f, v_{k}\right)=\sum(-1)^{n} a_{n}\left(f, D^{n} v_{k}\right), \\
\left(E(H) f, v_{k}\right) & =\left(\left(\sum a_{n} H^{n}\right) f, v_{k}\right)=\sum a_{n}\left(H^{n} f, v_{k}\right) \\
& =\sum a_{n}\left(f, H^{n} v_{k}\right)=\sum a_{n}(2 k+1)^{n}\left(f, v_{k}\right)=E(2 k+1)\left(f, v_{k}\right) .
\end{aligned}
$$

25. Order of magnitude of expansion coefficients. We will treat several special classes of functions.

25.1. The case of $L_{2}$. By the completeness of the set of the $v_{k}$ in $L_{2}(21.4)$ a function $f \in L_{2}$ has expansion coefficients $c_{k}$ such that

$$
\sum_{k=0}^{\infty}\left|c_{k}\right|^{2}=(f, f)
$$

(Parseval).

The expansion $\sum c_{k} v_{k}$ of $f$ will converge to $f$ in the mean on $(-\infty, \infty)$. Conversely the Riesz-Fischer theorem [21, p. 27] asserts that a formal series $\sum c_{k} v_{k}$ is the expansion of a function $f \in L_{2}$ whenever the series $\sum\left|c_{k}\right|^{2}$ converges. 
25.2. Lemma. Let $f \in A$ have the expansion $\sum c_{k} v_{k}$. Then the expansion coeffcients $c_{\boldsymbol{k}}^{(n)}$ of $\left\{x^{n}\right\} f$ satisfy the inequality

$$
\begin{aligned}
\left|c_{k}^{(n)}\right| \leqq & \{(k+n) \cdots(k+1)\}^{1 / 2} \\
& \cdot 2^{-n / 2}\left(\left|c_{k+n}\right|+\left(\begin{array}{c}
n \\
1
\end{array}\right)\left|c_{k+n-2}\right|+\cdots+\left|c_{k-n}\right|\right) \\
\leqq & \{(k+n) \cdots(k+1)\}^{1 / 2} 2^{n / 2} \max \left(\left|c_{k+n}\right|, \cdots,\left|c_{k-n}\right|\right) .
\end{aligned}
$$

The proof is obtained from (23.5) by induction with respect to $n$.

25.3. The case $\{|x|+1\}^{-n} f \in L_{2}$. Let $f$ be of the form indicated here with $n$ an integer $\geqq 0$. Then we may write $f=\{x+i\}^{n} f_{0}$ where $f_{0} \in L_{2}$. Set $f \sim \sum c_{k} v_{k}$, $f_{0} \sim \sum d_{k} v_{k}$. By 25.2

$$
\left|c_{k}\right| \leqq M_{n}(k+1)^{n / 2} \max \left(\left|d_{k+n}\right|, \cdots,\left|d_{k-n}\right|\right), \quad k=0,1, \cdots
$$

Hence by 25.1 the series

$$
\sum_{k=0}^{\infty}\left|c_{k}\right|^{2} /(k+1)^{n}
$$

converges.

For a related case see 32.3. We note the following corollary.

25.4. The case of $L_{1}$. Let $f$ be in $L_{1}, f \sim \sum c_{k} v_{k}$. Then there are constants $M$ and $p, p \leqq 1$, such that

$$
\left|c_{k}\right| \leqq M(k+1)^{p}, \quad k=0,1, \cdots .
$$

For an inequality of this kind with $p \leqq 1 / 2$ will hold for the expansion coefficients of the bounded function $\int_{0}^{x} f(25.3)$. The result now follows by applying the operator $D(24.1)$.

By a more straightforward argument $c_{k}=\left(f, v_{k}\right)$ is bounded by a constant times the maximum of $\left|v_{k}(x)\right|,-\infty<x<\infty$. Hence by $[17$, p. 236] the above inequality for the $c_{k}$ holds with $p=-1 / 12$.

25.5. The case of $A$. For $f \in A(21.2)$ one can write $f=E f_{0}$ where $E(x)=e^{a x^{2}}$ with an $a<1 / 2$ and $f_{0} \in L_{1}$. One may then use (23.7) to express the coefficients of $f$ in terms of those of $\{x\}^{n} f_{0}$. Subsequent application of 25.2 and 25.4 should lead to an estimate for the expansion coefficients of $f$. It turns out, however, that 25.2 is not sufficiently precise to give a convergent majorant for the coefficients of $f$ when $a$ exceeds the value $1 / 4$.

However, $\left(f, v_{k}\right)$ will be bounded by a constant times the maximum of $\left|e^{a x^{2}} v_{k}(x)\right|,-\infty<x<\infty$. Applying the estimate given in $[17$, p. 195] one obtains

$$
\left|\left(f, v_{k}\right)\right| \leqq M m^{k}, \quad k=0,1, \cdots
$$

where $m$ may be any number greater than $(1+2 a)^{1 / 2} /(1-2 a)^{1 / 2}$. 
Lemma 25.2 is sufficiently precise to deal with the class of functions of exponential growth. However, the representation obtained for these functions in 35 automatically gives an estimate for their expansion coefficients.

\section{REPRESENTATION OF FUNCTIONS OF POLYNOMIAL AND EXPONENTIAL GROWTH}

31. The differential equation $(H+p) y=f$. We will always take $p$ real and $\geqq 0$, and start by considering the homogeneous equation

$$
(H+p) y \equiv-y^{\prime \prime}+\left(x^{2}+p\right) y=0, \quad-\infty<x<\infty .
$$

The Liouville transformation $[1$, p. 109]

$$
t=\int_{0}^{x}\left(u^{2}+p\right)^{1 / 2} d u, \quad z(t)=\left(x^{2}+p\right)^{1 / 4} y(x), \quad(x \geqq 0)
$$

transforms (31.1) into

$$
\frac{d^{2} z}{d t^{2}}-\left\{1-\frac{3 x^{2}-2 p}{4\left(x^{2}+p\right)^{3}}\right\} z=0 .
$$

The latter equation possesses solutions which together with their first order derivatives behave as $c \exp ( \pm t)$ as $t \rightarrow \infty[1, \mathrm{p} .126, \mathrm{p} .60]$.

Returning to (31.1) let $w(x)$ be a solution which tends to 0 as $x \rightarrow \infty$, and which is such that $w\left(x_{0}\right)>0$ for some $x_{0}$. Then $w(x)>0$ for all $x$. We first prove this for $x>x_{0}$. Suppose that $x_{1}$ is the smallest number $>x_{0}$ such that $w\left(x_{1}\right)=0$. Then $w^{\prime}\left(x_{1}\right) \leqq 0$, and since $w^{\prime}\left(x_{1}\right) \neq 0$ (or $w$ would be the zero solution) we have $w^{\prime}\left(x_{1}\right)<0$. It follows that there is an interval $\left(x_{1}, x_{2}\right)$ on which $w(x)<0$. Then by $(31.1) w^{\prime \prime}(x) \leqq 0$ on $\left(x_{1}, x_{2}\right)$, hence $w^{\prime}(x) \leqq w^{\prime}\left(x_{1}\right)$, and thus $w$ continues to decrease. This contradicts the assumption that $w(x) \rightarrow 0$ as $x \rightarrow \infty$. Since the graph of $w(x)$ remains convex as long as $w(x)>0$ it is impossible for $w(x)$ to become equal to 0 for $x<x_{0}$. It follows actually that $w(x) \rightarrow \infty$ as $x \rightarrow-\infty$. One has $w^{\prime}(x)<0$ for all $x$.

The functions $w(x), w(-x)$ form a pair of linearly independent solutions of (31.1). The Wronskian $-w(x) w^{\prime}(-x)-w(-x) w^{\prime}(x)$ of these two solutions is constant since there is no $y^{\prime}$ term in the differential equation $[4$, p. 83]. The Wronskian is a positive constant. It is no restriction to assume that it has the value 1 .

One has as a first approximation

$$
w(x) \sim c( \pm) \cdot\left(x^{2}+p\right)^{-1 / 4} \exp \left\{-\int_{0}^{x}\left(u^{2}+p\right)^{1 / 2} d u\right\}, \quad x \rightarrow \pm \infty .
$$

Corresponding estimates for $w^{\prime}(x)$ may be obtained by formal differentiation of (31.2).

31.3. TheOREm. Let $w(x)$ be the positive solution of (31.1) such that $w(x) \rightarrow 0$ 
as $x \rightarrow \infty$ and with the normalization $w(0) w^{\prime}(0)=-1 / 2$. Let $f \in A(21.2)$. Then the differential equation

$$
(H+p) y=f
$$

possesses exactly one solution $\tilde{f} \in A$. It is given by the formula

$$
\tilde{f}(x)=w(x) \int_{-\infty}^{x} f(u) w(-u) d u+w(-x) \int_{x}^{\infty} f(u) w(u) d u .
$$

Proof. By (31.2)

$$
|w(x)| \leqq\left\{\begin{array}{lr}
C x^{-p / 2-1 / 2} e^{-x^{2} / 2}, & x \rightarrow \infty, \\
C|x|^{p / 2-1 / 2} e^{x^{2} / 2}, & x \rightarrow-\infty .
\end{array}\right.
$$

Hence the integrals in (31.5) exist. Simple estimates based on (31.6) show that $\tilde{f}$ as defined in (31.5) is of class $A$.

Since the Wronskian of $w(x)$ and $w(-x)$ has the value 1 the general solution of (31.4) is of the form $[4$, p. 87]

$$
\tilde{f}(x)+c_{1} w(x)+c_{2} w(-x)
$$

which is of class $A$ only if $c_{1}=c_{2}=0$.

32. Functions of polynomial growth. A function $f$ defined on $(-\infty, \infty)$ is said to be of (at most) polynomial growth if it is locally integrable and if there exist constants $M, m$ such that

$$
|f(x)| \leqq M\left(x^{2}+1\right)^{m}, \quad-\infty<x<\infty .
$$

32.2. LemMA. Let $f$ be of polynomial growth and let $M, m$ be such that (32.1) holds. Let $\tilde{f}$ be the unique function of class $A$ such that $(H+p) \tilde{f}=f(p \geqq 0 ; 31.3)$. Then there is a constant $N$ depending only on $M, m$ and $p$ such that

$$
|\tilde{f}(x)| \leqq N\left(x^{2}+1\right)^{m-1}, \quad-\infty<x<\infty .
$$

Proof. The function $\tilde{f}$ is given by (31.5) where $w$ satisfies the inequalities (31.6). The first term on the right hand side of (31.5) is bounded by

$$
\begin{gathered}
C_{1} x^{-p / 2-1 / 2} e^{-x^{2} / 2}\left(\int_{-\infty}^{1}|f(u)| w(-u) d u+\int_{1}^{x} u^{2 m+p / 2-3 / 2} d e^{u^{2} / 2}\right) \\
\leqq C_{2} x^{-p / 2-1 / 2} e^{-x^{2} / 2} \cdot x^{2 m+p / 2-3 / 2} e^{x^{2} / 2}, \quad x>1, \\
C_{3}|x|^{p / 2-1 / 2} e^{x^{2} / 2} \int_{-\infty}^{x}|u|^{2 m-p / 2-3 / 2}\left|d e^{-u^{2} / 2}\right| \\
\leqq C_{4}|x|^{p / 2-1 / 2} e^{x^{2} / 2} \cdot|x|^{2 m-p / 2-3 / 2} e^{-x^{2} / 2}, \quad x<-1 .
\end{gathered}
$$

A similar pair of estimates for the second term on the right hand side of (31.5) completes the proof. 
32.3. THEOREM. Let $f$ be a function of polynomial growth and let $M, m$ be such that (32.1) holds. Then $f$ may be written in the form

$$
f=H^{n} f_{0}
$$

for any integer $n \geqq 0$ and $>m+1 / 2$ and a corresponding function $f_{0} \in L_{2}$. The Hermite expansion $\sum c_{k} v_{k}$ of $f$ is such that for any $n$ as above there is a constant $R$ such that

$$
\left|c_{k}\right| \leqq R(2 k)^{n}, \quad k=1,2, \cdots .
$$

Proof. Repeated application of 32.2 with $p=0$ proves the representation $f=H^{n} f_{0}$. By 25.1 the expansion coefficients $d_{k}$ of the function $f_{0} \in L_{2}$ are bounded, while by (24.4) the expansion coefficients of $f$ are of the form $c_{k}=(2 k+1)^{n} d_{k}$.

33. Functions of exponential growth. A function $f$ defined on $(-\infty, \infty)$ is said to be of (at most) exponential growth if it is locally integrable and if there are constants $C, c$ such that

$$
|f(x)| \leqq C e^{c|x|}, \quad-\infty<x<\infty .
$$

We define the type of the function $f$ of exponential growth as the number $m$ given by

$$
m=\max (\mu, 0), \quad \mu=\limsup _{|x| \rightarrow \infty} \frac{\log |f(x)|}{|x|} .
$$

It will be necessary for us to construct a majorant of $|f(x)|$ with certain special properties.

33.3. Theorem. Let $f$ be of exponential type $m$. There exists a majorant of $|f(x)|$ of the form

$$
E_{0}\left(x^{2}\right) \text { where } E_{0}(z)=c \prod_{j}\left(1+z / p_{j}\right),
$$

the $p_{j}$ are real, $>0$ and such that the infinite series $\sum 1 / p_{j}$ converges, while $E_{0}\left(x^{2}\right)$ is of the same exponential type $m$ as $f$.

Proof. It is no restriction to assume that $|f(x)|<1$ for $|x|<1$. Set

$$
\sup _{|x| \geqq n-1} \frac{\log |f(x)|}{|x|}=\mu_{n}, \quad n=1,2, \cdots,
$$

and define

$$
m_{n}=\max \left(\mu_{n}, m+1 / n\right) .
$$

Since $\mu_{n} \downarrow \mu$ one concludes that $m_{n} \downarrow m$ as $n \rightarrow \infty$. The function $2 \cosh m_{n} x$ will be a strict majorant of $|f(x)|$ for $|x| \geqq n-1$. We denote the infinite product for $2 \cosh m_{n} x$ by $\pi_{n}$. 
Let $\pi_{11}$ be the partial product of $\pi_{1}$ of lowest order $\geqq 1$ which strictly majorizes $|f(x)|$ on $(-1,1)$. Let $\nu_{1}$ denote the number of factors of $\pi_{11}$, and let $\pi_{2}{ }^{*}$ denote the product obtained from $\pi_{2}$ by omitting the first $\nu_{1}$ factors. Since the factors of $\pi_{2}$ are smaller than those of $\pi_{1}$ but greater than 1 one has for all $x$

$$
|f(x)| \leqq \pi_{11} \pi_{2}^{*} \leqq \pi_{1} .
$$

Now let $\pi_{22}$ be the partial product of $\pi_{11} \pi_{2}{ }^{*}$ of lowest order $\geqq 2$ which strictly majorizes $|f(x)|$ on $(-2,2)$. Let $\nu_{2}$ denote the number of factors of $\pi_{22}$, and let $\pi_{3}{ }^{*}$ denote the product obtained from $\pi_{3}$ by omitting the first $\nu_{2}$ factors. Note that $\nu_{2} \geqq \nu_{1}$. Then for all $x$

$$
|f(x)| \leqq \pi_{22} \pi_{3}^{*} \leqq \pi_{1} .
$$

Continue in this manner and define $E_{0}\left(x^{2}\right)=\lim \pi_{n n}(x)$. The limit exists since $\pi_{n n}$ is increasing $\left(\nu_{n} \geqq \nu_{n-1}\right)$ while $\pi_{n n} \leqq \pi_{1}$. Since $\pi_{n n}$ majorizes $|f(x)|$ on $(-n, n)$ the limit will majorize $|f(x)|$ on $(-\infty, \infty)$. Noting that $\pi_{n+1}^{*}$ consists of $\pi_{n+1}$ minus its first $\nu_{n}$ factors and hence $\rightarrow 1$ as $n \rightarrow \infty$ we find that $E_{0}\left(x^{2}\right)$ $=\lim \pi_{n n}(x) \pi_{n+1}^{*}(x)$. The sequence $\pi_{n n} \pi_{n+1}^{*}$ is decreasing. It follows that the type of $E_{0}\left(x^{2}\right)$ is no larger than the type of $\pi_{n n} \pi_{n+1}^{*}$. But $\pi_{n n}$ is a polynomial, hence the latter function has the same type as $\pi_{n+1}^{*}$, that is, its type is $m_{n+1}$. Thus $E_{0}\left(x^{2}\right)$ has type $\leqq m$. Since $E_{0}\left(x^{2}\right)$ majorizes $|f(x)|$ its type can not be less than $m$.

We note that $p_{j}$ is at least equal to the square of the absolute value of the $j$ th zero of $\cosh m_{1} z$ on the positive imaginary axis:

$$
\left|p_{j}\right| \geqq(j-1 / 2)^{2} \pi^{2} / m_{1}^{2}, \quad j=1,2, \cdots .
$$

33.6. Lemma. The majorant $E_{0}\left(x^{2}\right)$ in 33.3 may be chosen such that all the $p_{j}$ are larger than the number

$$
\alpha=\underset{-\infty<x<\infty}{\text { l.u.b. }}\left\{\left(\sum_{j} \frac{2 x}{x^{2}+p_{j}}\right)^{2}+\sum_{j} \frac{2}{x^{2}+p_{j}}\right\} .
$$

Proof. By (33.5) the quantity $\alpha$ is finite for the original majorant constructed in the proof of 33.3. It is as a matter of fact no larger than

$$
\text { l.u.b. }\left\{m_{1}^{2} \tanh ^{2} m_{1} x+\sum_{j} 2 m_{1}^{2} /(j-1 / 2)^{2} \pi^{2}\right\}=2 m_{1}^{2} \text {. }
$$

Now replace all original $p_{j}$ which are $\leqq \alpha$ by $\alpha+1$, increasing $c$ in (33.4) if necessary to ensure that the new $E_{0}\left(x^{2}\right)$ is still a majorant of $|f(x)|$. The new value of $\alpha$ will be no larger than the old one.

34. Once more the equation $(H+p) y=f$. In this section we will prove a number of lemmas which will be used in the proof of the representation theorem for functions of exponential growth (35). The first lemma is basic. 
34.1. Lemma. Let $f$ be of class $A$ (21.2) and such that

$$
|f(x)| \leqq g(x)=\left(x^{2}+p\right) h(x), \quad-\infty<x<\infty,
$$

where $p>0, h(x)>0, h \in I_{2} \cap A$ (11), while for some $\alpha<p$ one has $\left|h^{\prime \prime}(x)\right|$ $\leqq \alpha h(x),-\infty<x<\infty$. Then the unique function $\tilde{f}$ of class $A$ such that $(H+p) \tilde{f}$ $=f(31.3)$ satisfies the inequality

$$
|\tilde{f}(x)| \leqq \frac{1}{1-\alpha / p} h(x), \quad-\infty<x<\infty .
$$

Proof. By (31.5) and the positivity of $w$ the solution $\tilde{f}$ is majorized by the solution $\tilde{g}$ in $A$ of the equation $(H+p) \tilde{g}=g$. Using the fact that $\left(x^{2}+p\right) w(x)$ $=w^{\prime \prime}(x)$ one obtains from (31.5) that

$$
\tilde{g}(x)=w(x) \int_{-\infty}^{x} h(u) w^{\prime \prime}(-u) d u+w(-x) \int_{x}^{\infty} h(u) w^{\prime \prime}(u) d u .
$$

Integration by parts transforms the right hand side to

$$
h(x)+w(x) \int_{-\infty}^{x} h^{\prime \prime}(u) w(-u) d u+w(-x) \int_{x}^{\infty} h^{\prime \prime}(u) w(u) d u .
$$

Here we have used the facts that the Wronskian $-w(x) w^{\prime}(-x)-w(-x) w^{\prime}(x)$ has the value 1 and that $w(x)$ as well as $w^{\prime}(x)$ are so small at $+\infty$ that the contributions of the integrated terms at $\pm \infty$ vanish (compare (31.2); since $h^{\prime \prime} \in A$ the functions $h$ and $h^{\prime}$ are dominated by expressions of the form $C \exp \left(b x^{2}\right)$ with $\left.b<1 / 2\right)$.

We finally use the inequality for $\left|h^{\prime \prime}\right|$ to replace $h^{\prime \prime}$ in the last two integrals by the majorant $(\alpha / p) g$. Thus

$$
\tilde{g}(x) \leqq h(x)+(\alpha / p) \tilde{g}(x)
$$

and the result follows.

34.2. Lemma. Let $f$ be locally integrable and let $|f(x)|$ be majorized by $E_{0}\left(x^{2}\right)$ where $E_{0}(z)=c \prod_{j}\left(1+z / p_{j}\right)$ is an infinite product with the properties of 33.3 and 33.6. Let $g_{0}=f$ and let $g_{n}$ denote the unique function in $A$ such that

$$
\left(1+H / p_{n}\right) g_{n}=g_{n-1}, \quad n=1,2, \cdots \text {. }
$$

Then

$$
\begin{aligned}
\left|g_{n}(x)\right| & \leqq c \prod_{j \leqq n} \frac{1}{1-\alpha / p_{j}} \prod_{j>n}\left(1+x^{2} / p_{j}\right) \\
& \leqq\left\{\prod_{j} \frac{1}{1-\alpha / p_{j}}\right\} E_{0}\left(x^{2}\right), \quad-\infty<x<\infty, n=0,1, \cdots .
\end{aligned}
$$


Proof. (34.3) is true for $n=0$. Suppose it has been proved already with $n$ replaced by $n-1$ where $n-1 \geqq 0$. That is,

where

$$
\left|p_{n} g_{n-1}(x)\right| \leqq\left(x^{2}+p_{n}\right) h_{n}(x)
$$

$$
h_{n}(x)=C_{n} \prod_{j>n}\left(1+x^{2} / p_{j}\right), \quad C_{n}=c \prod_{j \leq n-1} \frac{1}{1-\alpha / p_{j}} .
$$

One has

$$
\frac{h^{\prime}}{h}=\sum_{j>n} \frac{2 x}{x^{2}+p_{j}}
$$

hence by (33.7)

$$
\left|\frac{h^{\prime \prime}}{h}\right|=\left|\left(\frac{h^{\prime}}{h}\right)^{2}+\sum_{j>n} \frac{2 p_{j}-2 x^{2}}{\left(x^{2}+p_{j}\right)^{2}}\right| \leqq \alpha, \quad-\infty<x<\infty .
$$

Application of 34.1 with $p=p_{n}, h=h_{n}, f=p_{n} g_{n-1}$ therefore shows that the solution $g_{n}$ in $A$ of $\left(H+p_{n}\right) g_{n}=p_{n} g_{n-1}$ satisfies (34.3).

34.3. Lemma. Let $f, E_{0}$ and $g_{n}$ be as in 34.2. The sequence of functions $\left\{g_{n}\right\}$ is uniformly convergent on every finite interval. The limit function $g$ is bounded on $(-\infty, \infty)$. It is infinitely differentiable, $H^{r} g_{n} \rightarrow H^{r} g$ uniformly on every finite interval and $H^{r} g$ is of class $A$ for every integer $r \geqq 0$.

Proof. For $n \geqq 1(34.2)$

$$
H g_{n}=p_{n}\left(g_{n-1}-g_{n}\right) \text {. }
$$

Thus $H g_{n} \in A$ and by the definition of $g_{n}$ one may describe $H g_{n}$ as the unique function of $A$ such that

$$
\left(1+H / p_{n}\right) H g_{n}=H g_{n-1}, \quad n=2,3, \cdots .
$$

We may now apply 34.2 with $f$ replaced by $H g_{1}$. Since $H g_{1}=p_{1}\left(g_{0}-g_{1}\right)$ is majorized by $2 p_{1} E_{0}\left(x^{2}\right) /\left(1-\alpha / p_{1}\right)$ (34.3) one concludes from 34.2 that all functions $H g_{n}$ are majorized by

$$
\Omega(x)=2 p_{1} E_{0}\left(x^{2}\right) \prod_{j} 1 /\left(1-\alpha / p_{j}\right) .
$$

Hence by (34.5)

$$
\left|g_{n}(x)-g_{n-1}(x)\right| \leqq \Omega(x) / p_{n}, \quad-\infty<x<\infty, n=1,2, \cdots .
$$

We conclude that the sequence $g_{n}=g_{0}+\left(g_{1}-g_{0}\right)+\cdots+\left(g_{n}-g_{n-1}\right)$, $n=1,2, \cdots$ is uniformly convergent on every finite interval. By (34.3) the limit function $g$ will be bounded by the constant $c \prod_{j} 1 /\left(1-\alpha / p_{j}\right)$.

By a similar argument the sequence $\left\{H g_{n}\right\}$ is uniformly convergent on every finite interval. Since $\left\{x^{2}\right\} g_{n} \rightarrow\left\{x^{2}\right\} g$ it follows that the sequence $D^{2} g_{n}$ is 
uniformly convergent on every finite interval. Calling the limit function $g^{*}$ one concludes that $g_{n} \rightarrow \iint g^{*}$, hence $g=\iint g^{*}$ is of class $I_{2}$ (11). Furthermore $D^{2} g=g^{*}$ and $H g_{n} \rightarrow\left\{x^{2}\right\} g-g^{*}=H g$. Since the $H g_{n}$ are majorized by a fixed function of class $A$ the limit $H g$ is in $A$. One next proves that $H^{2} g_{n} \rightarrow H^{2} g$ and that $H^{2} g \in A$, etc.

35. Representation of functions of exponential growth.

35.1. ThEOREM. Let $f$ be a function of exponential growth, and let $m$ be the type of $f$. Let $E_{0}\left(x^{2}\right)$ be a majorant of $|f(x)|$ of type $m$ such that $E_{0}$ is an infinite product with the properties stated in 33.3 and 33.6. Then there is an infinitely differentiable function $f_{0} \in L_{2}$ such that

$$
f=H \cdot E_{0}(H) f_{0}
$$

in the sense that

$$
f=H f^{*}, \quad f^{*}=\lim _{n \rightarrow \infty} f_{n}, \quad f_{n}=c \prod_{j \leq n}\left(1+H / p_{j}\right) f_{0} .
$$

The Hermite expansion $\sum c_{k} v_{k}$ of $f$ is such that for every number $b>m$ there is $a$ constant $M$ such that

$$
\left|c_{k}\right| \leqq M \exp \left\{b(2 k)^{1 / 2}\right\}, \quad k=0,1, \cdots .
$$

Proof. Let $g_{0}=f$ and let $g_{r}$ be the unique function of $A$ such that $\left(1+H / p_{r}\right) g_{r}=g_{r-1}, r=1,2, \cdots$. By 34.2 the $g_{r}$ are majorized by a fixed constant multiple of $E_{0}\left(x^{2}\right)$, and by 34.4 they converge to a bounded infinitely differentiable function $g$ as $r \rightarrow \infty$. Moreover $H^{n} g_{r} \rightarrow H^{n} g$ as $r \rightarrow \infty$ and $H^{n} g \in A, n=1,2, \cdots$.

We define $f_{0}$ as the unique function of $A$ such that

$$
c H f_{0}=g \text {. }
$$

Since $g$ is bounded $f_{0}$ will be bounded and in $L_{2}(32.2)$. Moreover $f_{0}$ will be infinitely differentiable because $g$ is: $D^{2} f_{0}=\left\{x^{2}\right\} f_{0}-g / c$. By the above $H^{n} f_{0} \in A, n=0,1, \cdots$.

We next define

$$
f_{n}=c \prod_{j \leq n}\left(1+H / p_{j}\right) f_{0}, \quad n=1,2, \cdots
$$

Clearly $f_{n} \in A$.

In order to prove the convergence of the $f_{n}$ we write

$$
H f_{n}=\lim _{r \rightarrow \infty} g_{n r}, \quad g_{n r}=\prod_{j \leqq n}\left(1+H / p_{j}\right) g_{r} \quad(r \geqq n) .
$$

Since $g_{r} \in I_{2 r}$ (11) we have $g_{n r} \in I_{2 r-2 n}$. Because $H^{n} g_{r}$ is a linear combination of $g_{r}, \cdots, g_{r-n}$ we have $g_{n r} \in A$. It therefore follows from the definition of the $g_{r}$ that for fixed $n$ the functions $g_{n r}$ may be described as the unique functions of $A$ such that 


$$
\left(1+H / p_{r}\right) g_{n r}=g_{n, r-1}, \quad r>n ; \quad g_{n n}=g_{0}=f .
$$

Application of 34.2 shows that the functions $\left|g_{n r}(x)\right|$ are majorized by $\left\{\prod_{j>n} 1 /\left(1-\alpha / p_{j}\right)\right\} E_{0}\left(x^{2}\right), r=n, n+1, \cdots$. Letting $r \rightarrow \infty$ it follows that

By (35.3)

$$
\left|H f_{n}(x)\right| \leqq\left\{\prod_{j>n} 1 /\left(1-\alpha / p_{j}\right)\right\} E_{0}\left(x^{2}\right), \quad n=1,2, \cdots
$$

$$
f_{n}-f_{n-1}=H f_{n-1} / p_{n}, \quad n=1,2, \cdots .
$$

Hence by (35.4) and the convergence of $\sum 1 / p_{n}$ the sequence $f_{n}=f_{1}+\left(f_{2}-f_{1}\right)$ $+\cdots+\left(f_{n}-f_{n-1}\right)$ is uniformly convergent on every finite interval. Moreover since $f_{1}$ is bounded (35.4) implies that

$$
\left|f_{n}(x)\right| \leqq\left|f_{1}(x)\right|+\sum_{j \geqq 1}\left|H f_{j}(x)\right| / p_{j+1} \leqq C E_{0}\left(x^{2}\right), \quad n=1,2, \cdots
$$

It follows that the limit function $f^{*}$ of the $f_{n}$ is of class $A$.

We will next show that $f=H f^{*}$. Let $f$ have the Hermite expansion $\sum c_{k} v_{k}$. Then by (24.4) and the definition of $g_{r}$

$$
\left(g_{r}, v_{k}\right)=c_{k} / \prod_{j \leqq r}\left\{1+(2 k+1) / p_{j}\right\} .
$$

Now the $g_{r}$ are all majorized by the same multiple of $E_{0}\left(x^{2}\right)$. Hence by dominated convergence

$$
\left(g, v_{k}\right)=\lim _{r \rightarrow \infty}\left(g_{r}, v_{k}\right)=c_{k} / \prod_{j}\left\{1+(2 k+1) / p_{j}\right\}=c_{k} c / E_{0}(2 k+1) .
$$

Thus $f_{0} \sim \sum d_{k} v_{k}$ where by (35.2) and (24.4)

$$
d_{k}=\frac{c_{k}}{(2 k+1) E_{0}(2 k+1)} .
$$

It next follows from (35.3) that

$$
\left(f_{n}, v_{k}\right)=c_{k} /(2 k+1) \prod_{j>n}\left\{1+(2 k+1) / p_{j}\right\},
$$

and hence by dominated convergence (35.5) that

$$
\left(f^{*}, v_{k}\right)=\lim _{n \rightarrow \infty}\left(f_{n}, v_{k}\right)=c_{k} /(2 k+1) .
$$

However, these numbers are also the expansion coefficients of the function $\tilde{f}$ of $A$ such that $H \tilde{f}=f(24.4)$. Since both $f^{*}$ and $\tilde{f}$ are in $A$ we conclude that $f^{*}=\tilde{f}(23.4)$ and hence $f=H f^{*}$.

We finally estimate the expansion coefficients $c_{k}$ of $f$. Since $f_{0} \in L_{2}$ its expansion coefficients $d_{k}$ are bounded. Thus by (35.6) 


$$
\left|c_{k}\right| \leqq K(2 k+1) E_{0}(2 k+1)
$$

( $K$ a constant), and the desired estimate follows because $E_{0}\left(x^{2}\right)$ is of exponential type $m$.

\section{FOURIER TRANSFORM THEORY FOR $L_{1}$}

41. The inversion formula. For $f \in L_{1}$ the Fourier transform $g=T f$ is defined by

$$
g(x)=(T f)(x)=(2 \pi)^{-1 / 2} \int_{-\infty}^{\infty} f(u) e^{-i x u} d u,
$$

and the conjugate Fourier transform $h=\bar{T} f$ by

$$
h(x)=(\bar{T} f)(x)=(2 \pi)^{-1 / 2} \int_{-\infty}^{\infty} f(u) e^{i x \varkappa} d u .
$$

In general $g$ will not belong to $L_{1}$. However, $g$ will be a bounded continuous function. As a matter of fact, by the Riemann-Lebesgue lemma $g(x) \rightarrow 0$ as $x \rightarrow \pm \infty[19$, p. 11$]$.

For real $f$ the conjugate Fourier transform $h$ is the complex conjugate of the Fourier transform $g$. For even $f$ one has $h=g$.

41.3. Inversion theorem. Let $f \in L_{1}$, and let $g=T f$ also be in $L_{1}$. Then $\bar{T} g=f .($ Compare $[3$, p. $5 i]$.)

Proof. Let $f$ have the Hermite expansion $\sum c_{k} v_{k}$. Then by (24.2)

$$
g=T f \sim \sum(-i)^{k} c_{k} v_{k}
$$

and by (24.3)

$$
\bar{T} g \sim \sum i^{k}(-i)^{k} c_{k} v_{k}=\sum c_{k} v_{k}
$$

Thus $f$ and $\bar{T} g$ have the same Hermite expansion. Since both functions are of class $A$ they are identical (23.4).

41.4. Transform of convolution. Let $f_{1}, f_{2} \in L_{1}$. Then the convolution

$$
f(x)=\left(f_{1} * f_{2}\right)(x)=(2 \pi)^{-1 / 2} \int_{n}^{\infty} f_{1}(u) f_{2}(x-u) d u
$$

is in $L_{1}$, and

$$
T f=T f_{1} \cdot T f_{2} .
$$

That the convolution is in $L_{1}$ follows from Fubini's theorem, and the same theorem justifies the inversion of the order of integration which proves the formula for $T f$ (compare [19, p. 59]).

42. Application to uniqueness theorems. Using the inversion formula 41.3 we will strengthen the Uniqueness Theorem 23.4. 
42.1. The class $B$. A function $f$ is said to be of class $B$ if there is a real $b>0$ such that $f(x) \cdot \exp \left(-x^{2} / 2+b|x|\right)$ is integrable (and absolutely integrable) over $(-\infty, \infty)$.

42.2. UNIQUeNESS THEOREM. If two functions of $B$ have the same Hermite expansion they are equal.

Proof. Suppose that $f \in B$ has the Hermite expansion 0. Then

$$
\int_{-\infty}^{\infty} f(x) e^{-x^{2} / 2} x^{k} d x=0, \quad k=0,1, \cdots
$$

hence the function

$$
g(z)=\int_{-\infty}^{\infty} f(x) e^{-x^{2} / 2} e^{-i z x} d x
$$

which is analytic in some strip $|\operatorname{Im} z|<b$ vanishes at $z=0$ together with all its derivatives. Thus $g(z)=0$, that is, the Fourier transform of the function $f(x) \exp \left(-x^{2} / 2\right)$ of $L_{1}$ is equal to 0 . By 41.3 one concludes that $f=0$.

In the above proof the analyticity in the strip $|\operatorname{Im} z|<b$ may be replaced by quasi-analyticity on the real line.

42.4. Classes $Q$. Let $\left\{A_{k}\right\}$ be a nondecreasing sequence such that $\sum 1 / A_{k}$ diverges. A locally integrable function $f$ is said to be of class $Q\left\{A_{k}\right\}$ if

$$
\int_{-\infty}^{\infty}|f(x)| e^{-x^{2} / 2}|x|^{k} d x \leqq A_{k}^{k}, \quad k=0,1, \cdots
$$

42.5. Uniqueness THEOREM. If two functions of $Q\left\{A_{k}\right\}$ have the same Hermite expansion they are equal.

Proof. The function $g(z)$ defined in (42.3) will be quasi-analytic on $(-\infty, \infty)$ :

$$
\left|g^{(k)}(z)\right| \leqq A_{k}^{k}, \quad-\infty<z<\infty, k=0,1, \cdots .
$$

By hypothesis $g^{(k)}(0)=0, k=0,1, \cdots$. Thus since $\sum 1 / A_{k}$ diverges, Carleman's theorem [13, p. 14] implies that $g(z)=0$. Application of 41.3 completes the proof.

$$
\text { V. PANsions }
$$

51. Definitions. $A$ pansion $\phi$ is a formal series

$$
\sum_{k=0}^{\infty} c_{k} v_{k}
$$

where the $v_{k}$ are the normalized Hermite functions (21.1). The coefficients $c_{k}$ are real or complex numbers.

We define 


$$
\left(\phi, v_{k}\right)=c_{k}
$$

and will of ten write $\phi \sim \sum c_{k} v_{k}$ instead of $\phi=\sum c_{k} v_{k}$.

Two pansions are said to be equal if they have the same coefficients $c_{k}$.

The scalar multiple $a \phi$ and the sum $\phi+\psi$ of two pansions are defined by the corresponding operations on the coefficients.

If the pansion $\phi$ is the expansion of a function $f$ of class $A$ (21.2) or of the larger class $B(42.1)$ we identify $\phi$ with $f$. Instead of $B$ one could also use a fixed class $Q$ as defined in 42.4 .

52. Global differentiation $[D]$. Let $\phi$ be the pansion $\sum c_{k} v_{k}$. We define the global derivative $[D] \phi$ to be the pansion

$$
[D]_{\phi} \sim\left(\sum c_{k} D v_{k} \sim\right) \sum_{k=0}^{\infty}\left\{(k / 2+1 / 2)^{1 / 2} c_{k+1}-(k / 2)^{1 / 2} c_{k-1}\right\} v_{k}
$$

If $\phi$ is a function $f$ of class $I_{1}$ whose derivative $D f$ is of class $A$ or $B$ then $[D] \phi$ $=D f(24)$.

Using (22.2) one easily verifies that

$$
\left([D]_{\phi}, v_{k}\right)=-\left(\phi, D v_{k}\right) .
$$

Now let $f \in B$. By (52.2) the coefficients of the global $n$th derivative $[D]^{n} f$ are given by

$$
\left([D]^{n} f, v_{k}\right)=(-1)^{n}\left(f, D^{n} v_{k}\right) .
$$

Dirac's $\delta$. The $\delta$ "function" [5, p. 58] may be defined as the global derivative of the unit step function $U$ given by $U(x)=0, x \leqq 0, U(x)=1, x>0$. Thus

$$
\begin{aligned}
\left(\delta, v_{k}\right) & =\left([D] U, v_{k}\right)=-\left(U, D v_{k}\right) \\
& =-\int_{0}^{\infty}\left\{D v_{k}(x)\right\} d x=v_{k}(0),
\end{aligned}
$$

hence by (22.3)

$$
\delta=[D] U \sim \sum_{j=0}^{\infty} \frac{(-1)^{i}\{(2 j) !\}^{1 / 2}}{2^{i} j ! \pi^{1 / 4}} v_{2 j} .
$$

52.5. TheOREM. Every pansion is the global derivative of another pansion. The antiderivative is determined $u p$ to a constant function: $[D] \phi=0$ if and only if $\phi=\{c\}$, a constant function.

Proof. To obtain an antiderivative $\sum c_{k} v_{k}$ of a given pansion $\sum d_{k} v_{k}$ one has to solve the equations

$$
(k / 2+1 / 2)^{1 / 2} c_{k+1}-(k / 2)^{1 / 2} c_{k-1}=d_{k}, \quad k=0,1, \cdots
$$

(52.1). It is clear that $c_{0}$ can be chosen arbitrarily and that all other $c_{k}$ can be expressed in terms of $c_{0}$ and the $d$ 's. 
One has $[D]\{c\}=D\{c\}=0$. Now suppose that $[D] \phi=0$ where $\phi \sim \sum c_{k} v_{k}$. Solving the above equations with $d_{k}=0, k=0,1, \cdots$ one obtains $c_{1}=c_{3}$ $=c_{5}=\cdots=0$,

$$
c_{2 j}=\left(\frac{2 j-1}{2 j}\right)^{1 / 2} c_{2 j-2}=\cdots=\frac{\{(2 j) !\}^{1 / 2}}{2^{i j} !} c_{0} .
$$

Thus by 23.3 the pansion $\phi$ is the expansion of the constant function $\left\{2^{-1 / 2} \pi^{-1 / 4} c_{0}\right\}$, that is, $\phi$ is equal to that function.

52.6. Corollary. If two functions $f$ and $g$ of class $B$ (42.1) have equal global derivatives of order $n$ then $f$ and $g$ differ at most by a polynomial of degree $n-1$.

For $h=f-g$ satisfies $[D]^{n} h=0$. Hence by $52.5[D]^{n-1} h=\{c\}$, or

$$
[D]^{n-1}\left(h-\left\{c x^{n-1} /(n-1) !\right\}\right)=0 .
$$

Continuing one finds that $h$ is equal to a polynomial of degree $\leqq n-1$.

It follows from 52.6 that the Dirac $\delta$ can not be equal to a function $f$ (of class $B)$. For this would imply that $U(x)=\int_{0}^{x} f+c$ almost everywhere, hence $U(x)$ would differ from $c$ by less than $\epsilon=1 / 2$ for almost all $x$ on some interval $(-\gamma, \gamma)$.

53. Global multiplication by $\{x\}$. Let $\phi$ be the pansion $\sum c_{k} v_{k}$. We define the global product $[x] \phi$ to be the pansion

$$
[x]_{\phi} \sim\left(\sum c_{k}\{x\} v_{k} \sim\right) \sum_{k=0}^{\infty}\left\{(k / 2+1 / 2)^{1 / 2} c_{k+1}+(k / 2)^{1 / 2} c_{k-1}\right\} v_{k} .
$$

If $\phi$ is a function $f$ of class $B$ then $[x] \phi=\{x\} f$ (23.5). Using (22.2) one easily verifies that

$$
\left([x] \phi, v_{k}\right)=\left(\phi,\{x\} v_{k}\right) .
$$

53.3. Product rule. One has

$$
[D][x]_{\phi}=\phi+[x][D] \phi .
$$

For by (52.2) and (53.2)

$$
\begin{aligned}
\left([D][x] \phi, v_{k}\right) & =-\left([x] \phi, D v_{k}\right)=-\left(\phi,\{x\} D v_{k}\right) \\
& =-\left(\phi, D\{x\} v_{k}\right)+\left(\phi, v_{k}\right)=\left([x][D] \phi, v_{k}\right)+\left(\phi, v_{k}\right) .
\end{aligned}
$$

53.4. Corollary. If $\phi$ is the global nth derivative of a function $f \in B$ the same is true about $[x] \phi$.

For if $\phi=[D]^{n} f, f \in B$, then by the product rule 53.3

$$
[x][D]^{n} f=[D]^{n}\left(\{x\} f-n \int_{0}^{x} f\right) .
$$


53.5. Theorem. Every pansion can be written as $[x]$ times another pansion. The latter pansion is determined up to a constant multiple of the $\delta$ pansion: $[x] \phi=0$ if and only if $\phi$ is a constant multiple of $\delta$.

Proof. To write a given pansion $\sum d_{k} v_{k}$ as the product of $[x]$ and a pansion $\sum c_{k} v_{k}$ one has to solve the equations

$$
(k / 2+1 / 2)^{1 / 2} c_{k+1}+(k / 2)^{1 / 2} c_{k-1}=d_{k}, \quad k=0,1, \cdots
$$

(53.1). It is clear that $c_{0}$ can be chosen arbitrarily and that all other $c_{k}$ can be expressed in terms of $c_{0}$ and the $d$ 's.

A pansion $\phi \sim \sum c_{k} v_{k}$ satisfies $[x] \phi=0$ if and only if the $c_{k}$ satisfy the above equations with $d_{k}=0, k=0,1, \cdots$. That is, $c_{1}=c_{3}=c_{5}=\cdots=0$, and

$$
c_{2 j}=-\left(\frac{2 j-1}{2 j}\right)^{1 / 2} c_{2 j-2}=\cdots=(-1)^{j} \frac{\{(2 j) !\}^{1 / 2}}{2^{i j} !} c_{0} .
$$

Thus by (52.4) $\phi=\pi^{1 / 4} c_{0} \delta$.

54. $[\mathbf{H}]$ and other global operators. Let $\phi$ be the pansion $\sum c_{k} v_{k}$. Then we define

$$
[H]_{\phi} \sim\left(\sum c_{k} H v_{k} \sim\right) \sum_{k=0}^{\infty}(2 k+1) c_{k} v_{k} .
$$

Note that $[H]=[x]^{2}-[D]^{2}$. If $\phi$ is a function $f$ of class $I_{2}$ such that $D^{2} f$ is of class $A$ or $B$ then $[H] \phi=H f(24.4)$.

Let $E(x)=\sum a_{n} x^{n}$ be an entire function. Then we define (compare (24.6))

$$
[E(H)]_{\phi} \sim\left(\sum c_{k} E(H) v_{k} \sim\right) \sum_{k=0}^{\infty} E(2 k+1) c_{k} v_{k} .
$$

We define $[E(x)] \phi$ and $[E(D)] \phi$ by the equations (compare (23.7) and (24.5))

$$
\begin{aligned}
\left([E(x)]_{\phi}, v_{k}\right) & =\sum a_{n}\left([x]^{n} \phi, v_{k}\right), & & k=0,1, \cdots \\
\left([E(D)] \phi, v_{k}\right) & =\sum a_{n}\left([D]^{n} \phi, v_{k}\right), & k & =0,1, \ldots
\end{aligned}
$$

whenever the series on the right hand side are absolutely convergent. The following lemma gives some information about the applicability of definitions (54.3) and (54.4).

54.5. Lemma. Let $E(x)$ be an entire function of growth $\leqq$ order 2, type 0 . In other words, $E(x)=\sum a_{n} x^{n}$ where the $a_{n}$ satisfy an inequality

$$
\left|a_{n}\right| \leqq K(\epsilon)(n !)^{-1 / 2} \epsilon^{n}, \quad n=0,1, \cdots
$$

for every $\epsilon>0$ [2, p. 11]. Then definitions (54.3) and (54.4) are applicable to every pansion $\phi \sim \sum c_{k} v_{k}$ such that for some $m>0$ 


$$
\left|c_{k}\right| \leqq M m^{k}
$$$$
k=0,1, \cdots .
$$

The coefficients of $[E(x)] \phi$ and $[E(D)] \phi$ will satisfy a set of inequalities of the form (54.6) with a larger $m$.

When $\phi$ is a function $f$ of class $A$ then $[E(x)] \phi=\{E(x)\} f$.

Proof. Because of the similarity of the formulas for $[x]^{n} \phi$ and $[D]^{n} \phi$ it will be sufficient to prove (54.3). We may apply the proof of Lemma 25.2 to obtain the estimate

$$
\begin{aligned}
& \left|\left([x]^{n} \phi, v_{k}\right)\right| \\
& \leqq\{(k+n) \cdots(k+1)\}^{1 / 2} 2^{-n / 2}\left(\left|c_{k+n}\right|+C_{n, 1}\left|c_{k+n-2}\right|+\cdots+\left|c_{k-n}\right|\right) \\
& \leqq M_{1} 2^{(k+n) / 2}(n !)^{1 / 2} 2^{-n / 2} m^{k}(m+1 / m)^{n}, \\
&
\end{aligned}
$$

Hence by the given inequality for the $a_{n}$ the series in (54.3) will certainly converge if $(m+1 / m) \epsilon<1$. The sums of the series are bounded by $M_{2}\left(2^{1 / 2} m\right)^{k}$, $k=0,1, \cdots$. It is likely that a more precise lemma than 25.2 would show that $2^{1 / 2} m$ may be replaced by any number $\mu>m$ in this final estimate.

54.7. Corollary. Let $E_{1}, E_{2}$ be entire functions of growth $\leqq$ order 2 , type 0 , and let the coefficients of $\phi \sim \sum c_{k} v_{k}$ satisfy (54.6). Then

$$
\begin{aligned}
{\left[E_{1}(D)\right]\left[E_{2}(D)\right] \phi } & =\left[E_{1}(D) E_{2}(D)\right] \phi, \\
{\left[E_{1}(x)\right]\left[E_{2}(x)\right]_{\phi} } & =\left[E_{1}(x) E_{2}(x)\right] \phi .
\end{aligned}
$$

The crucial step in the proof is to show that $[D][E(D)] \phi=[D E(D)] \phi$. Set $[E(D)] \phi=\psi$. Writing for $D v_{k}$ the linear combination of $v_{k+1}$ and $v_{k-1}$ it is equal to one, has by (54.4)

$$
\left([D] \psi, v_{k}\right)=-\left(\psi, D v_{k}\right)=-\sum a_{n}\left([D]^{n} \phi, D v_{k}\right)=\sum a_{n}\left([D]^{n+1} \phi, v_{k}\right) .
$$

55. The translation operator $\left[e^{a D}\right]$. By 54.5 the operator $\left[e^{a D}\right]$ may be applied to any pansion $\phi \sim \sum c_{k} v_{k}$ whose coefficients satisfy an inequality (54.6). In particular $\left[e^{a D}\right]$ may be applied to any function $f$ of class $A$ (25.5). By (54.4) and (52.3)

$$
\begin{aligned}
\left(\left[e^{a D}\right] f, v_{k}\right) & =\sum a^{n}\left([D]^{n} f, v_{k}\right) / n ! \\
& =\sum(-a)^{n}\left(f, D^{n} v_{k}\right) / n !=\left(f, \sum(-a)^{n} D^{n} v_{k} / n !\right) \\
& =\left(f,\left\{v_{k}(x-a)\right\}\right) .
\end{aligned}
$$

The third step may be justified by dominated convergence. As a matter of fact

$$
\begin{aligned}
& \left|\frac{D^{n} v_{k}(x)}{n !}\right|=\left|\frac{1}{2 \pi} \int_{|z-x|=|2 a|} \frac{v_{k}(z)}{(z-x)^{n+1}} d z\right| \\
& \quad \leqq|2 a|^{-n} \max _{|z-x|=|2 a|}\left|v_{k}(z)\right| \leqq C|2 a|^{-n}(|x|+1)^{k} \exp \left(|2 a x|-x^{2} / 2\right),
\end{aligned}
$$


and hence the order of summation and integration may be inverted.

Now let $a$ be real for a moment. Then

$$
\left(f,\left\{v_{k}(x-a)\right\}\right)=\left(\{f(x+a)\}, v_{k}\right) .
$$

Thus we have the following result to justify the name translation operator.

55.1. Theorem. Let $f$ be a function of class $A$ and let a be real. Then

$$
\left[e^{a D}\right] f=\{f(x+a)\}
$$

(Taylor's formula!).

Another translation rule is as follows.

55.2. RULE. Let $E(x)$ be an entire function of growth $\leqq$ order 2 , type 0 , and let the coefficients of $\phi$ satisfy a set of inequalities (54.6). Then for real or complex $a$

$$
\left[e^{a D}\right][E(x)] \phi=[E(x+a)]\left[e^{a D}\right] \phi .
$$

Proof. The proof follows from the product rule 53.3 in the case $E(x)=x$. Now let $E(x)=\sum b_{n} x^{n},[E(x)] \phi=\psi$. The general case follows from the identity

$$
\begin{aligned}
\left([D]^{p} \psi, v_{k}\right) & =(-1)^{p}\left(\psi, D^{p} v_{k}\right)=(-1)^{p} \sum b_{n}\left([x]^{n} \phi, D^{p} v_{k}\right) \\
& =\sum b_{n}\left([D]^{p}[x]^{n} \phi, v_{k}\right)
\end{aligned}
$$

where $D^{p_{v_{k}}}$ denotes the linear combination of the $v_{j}$ it is equal to.

Multiplying by $a^{p} / p$ ! and summing over $p$ one obtains

$$
\left(\left[e^{a D}\right] \psi, v_{k}\right)=\sum_{n} b_{n}\left([x+a]^{n}\left[e^{a D}\right] \phi, v_{k}\right) .
$$

55.3. RuLE. Whenever the coefficients of $\phi$ satisfy (54.6) one has by 54.7

$$
\left[e^{a D}\right]\left[e^{b D}\right]_{\phi}=\left[e^{(a+b) D}\right]_{\phi} .
$$

55.4. Corollary. If $\left[e^{a D}\right] \phi=0$ then $\phi=0$.

55.5. Corollary. One has $[x-a] \phi=0$ if and only if

$$
\phi=c \delta_{a}=c\left[e^{a D}\right] \delta .
$$

Proof. One has by 55.2

$$
[x-a]_{\phi}=[x-a]\left[e^{-a D}\right]\left[e^{a D}\right]_{\phi}=\left[e^{-a D}\right][x]\left[e^{-a D}\right] \phi,
$$

hence the left hand side is equal to 0 if and only if $[x]\left[e^{a D}\right] \phi=0$. The result now follows from 53.5.

The preceding results have important counterparts obtained by interchanging $x$ and $D$. For example,

$$
\left[e^{a x}\right][E(D)] \phi=[E(D-a)]\left[e^{a x}\right] \phi,
$$

while $[D-a] \phi=0$ if and only if $\phi=c\left\{e^{a x}\right\}$. 
56. Global multiplication. We will begin by considering the expression

$$
\left(v_{r} v_{s}, v_{k}\right)=\alpha_{r s k} \text {. }
$$

Permutation of $r, s$ and $k$ does not change $\alpha_{r s k}$. We need the following estimate.

56.2. Lemma. For every $\epsilon>0$ there is a constant $C(s, k, \epsilon)$ such that

$$
\left|\alpha_{r s k}\right| \leqq C(s, k, \epsilon)\left(3^{-1 / 2}+\epsilon\right)^{r}, \quad r=0,1, \cdots .
$$

Proof. Using the generating function of the Hermite polynomials [17, p. 102] to obtain an integral representation for $v_{r}(x)$ one may write

$$
\begin{aligned}
\alpha_{r s k}= & \gamma_{1}(s, k)(r !)^{1 / 2} 2^{-r / 2} \int_{-\infty}^{\infty} H_{s}(x) H_{k}(x) \exp \left(-3 x^{2} / 2\right) d x \int_{|w|=1} w^{-r-1} \\
& \cdot \exp \left(2 x w-w^{2}\right) d w \\
= & \gamma_{1}(s, k)(r !)^{1 / 2} 2^{-r / 2} \int_{|w|=1} w^{-r-1} \exp \left(-w^{2} / 3\right) d w \int_{-\infty}^{\infty} p_{s+k}(x-2 w / 3) \\
& \cdot \exp \left\{-3 / 2(x-2 w / 3)^{2}\right\} d x .
\end{aligned}
$$

Here $H_{k}$ denotes the Hermite polynomial of degree $k$, and $p_{s+k}$ is a polynomial of degree $s+k$ with coefficients which depend on $s$ and $k$ and are polynomials in $w$ of degree $\leqq s+k$. Thus the last integral over $(-\infty, \infty)$ is equal to a polynomial in $w$ of degree $\leqq s+k$ with coefficients depending only on $s$ and $k$. It follows that $\alpha_{r s k}$ is equal to a finite sum of terms of the form

$$
C_{j}(s, k)(r !)^{1 / 2} 2^{-r / 2} 3^{-i} / j !
$$

where $j$ runs from $(r-s-k) / 2$ to $r / 2$. This proves the result.

56.3. Global product $\phi v_{k}$. Let $\phi \sim \sum c_{r} v_{r}$ where the $c$ 's satisfy an inequality (54.6) with $m<3^{1 / 2}$. Then we define the pansion $\phi v_{k}$ by

$$
\left(\phi v_{k}, v_{s}\right)=\sum_{r} c_{r}\left(v_{r} v_{k}, v_{s}\right)=\sum_{r} c_{r} \alpha_{r s k}, \quad s=0,1, \cdots
$$

We have to prove that the new definition is consistent with the usual definition if $\phi$ is a function $f$. Suppose that $f(x) \exp \left(-a x^{2}\right)=f_{1}(x)$ is in $L_{1}$ where $a<1 / 4$. Then by 25.5 the expansion coefficients $c_{r}$ of $f$ satisfy an inequality (54.6) with $m<3^{1 / 2}$. We have to show that in this case $\sum c_{r} \alpha_{r s k}$ $=\left(f v_{k}, v_{s}\right)$. One has by 56.2

$$
\begin{aligned}
\left|f(x) \alpha_{r s k} v_{r}(x)\right| & \leqq\left|f_{1}(x)\right| C_{1}\left(3^{-1 / 2}+\epsilon\right)^{r} \max \mid e^{a x^{2} v_{r}(x) \mid} \\
& \leqq C_{2}\left|f_{1}(x)\right|\left(3^{-1 / 2}+\epsilon\right)^{r} m^{r}, \quad r=0,1, \cdots
\end{aligned}
$$

where $m<3^{1 / 2}(25.5)$. It follows that the series $\sum_{r} f \alpha_{r s k} v_{r}$ is dominatedly convergent on $(-\infty, \infty)$, hence 


$$
\int_{-\infty}^{\infty}\left(\sum_{r} f \alpha_{r s k} v_{r}\right) d x=\sum_{r} c_{r} \alpha_{r s k}
$$

To evaluate the integral we remark that the series $\sum_{r} \alpha_{r s k} v_{r}$ is dominatedly convergent. Hence the expansion coefficients of its sum which is of class $A$ are the numbers $\alpha_{r s k}, r=0,1, \ldots$. But these are the expansion coefficients of $v_{s} v_{k}$. One concludes that $\sum_{r} \alpha_{r s k} v_{r}=v_{s} v_{k}$ and thus the above integral has the value $\left(f v_{k}, v_{s}\right)$ :

56.4. Lemma. Let $f$ be a function such that for some $a<1 / 4$ the product $f(x) \exp \left(-a x^{2}\right)$ is in $L_{1}$. Let $f \sim \sum c_{r} v_{r}$. Then

$$
\sum_{r} c_{r} \alpha_{r s k}=\left(f v_{k}, v_{s}\right)
$$

56.5. Global product $\phi \psi$. We defined $[x] \psi$ such that $\left([x] \psi, v_{k}\right)=\left(\psi,\{x\} v_{k}\right)$ where $\{x\} v_{k}$ stands for the linear combination of the $v_{s}$ it is equal to. For $\phi \sim \sum c_{r} v_{r}, \psi \sim \sum d_{s} v_{s}$ we similarly define the pansion $\phi \psi$ by

$$
\begin{aligned}
\left(\phi \psi, v_{k}\right) & =\left(\psi, \phi v_{k}\right)=\left(\psi, \sum_{s}\left(\sum_{r} \bar{c}_{r} \alpha_{r s k}\right) v_{s}\right) & \\
& =\sum_{s} d_{s} \sum_{r} c_{r} \alpha_{r s k}, & k=0,1, \cdots
\end{aligned}
$$

whenever the latter series converge.

One has in particular $1 \cdot \psi=\psi$. However, $\psi \cdot 1$ does not have to exist. The above multiplication is not commutative, nor associative. When $\phi$ is an entire function $E$ of growth $\leqq$ order 2, type 0 the above definition agrees with (54.3).

57. Convolution. We first consider the expression

$$
\left(v_{r} * v_{s}, v_{k}\right)=\beta_{r s k} .
$$

57.2. Lemma. One has

$$
\beta_{r s k}=i^{k}(-i)^{r+8} \alpha_{r s k}=(-1)^{(r+8-k) / 2} \alpha_{r s k} .
$$

Proof. By 41.4 and (22.4)

$$
T\left(v_{r} * v_{8}\right)=T v_{r} \cdot T v_{s}=(-i)^{r+s} v_{r} v_{s},
$$

hence by 41.3

$$
v_{r} * v_{s}=\bar{T}(-i)^{r+s} v_{r} v_{s}
$$

Thus by 22.4

$$
\beta_{r s k}=i^{k}(-i)^{r+s}\left(v_{r} v_{s}, v_{k}\right)=(-1)^{(r+s-k) / 2} \alpha_{r s k}
$$

since $\alpha_{r s k} \neq 0$ only if $r+s$ and $k$ have the same parity.

57.3. Convolution $\phi * v_{k}$. Let $\phi \sim \sum c_{r} v_{r}$ where the c's satisfy an inequality (54.6) with $m<3^{1 / 2}$. Then we define the pansion $\phi * v_{k}$ by 


$$
\left(\phi * v_{k}, v_{s}\right)=\sum_{r} c_{r}\left(v_{r} * v_{k}, v_{s}\right)=\sum_{r} c_{r} \beta_{r k s}, \quad s=0,1, \cdots
$$

This definition agrees with the usual definition 41.4 if $\phi$ is a function $f$ :

57.4. Lemma. Let $f$ be a function such that for some $a<1 / 4$ the product $f(x) \exp \left(-a x^{2}\right)$ is in $L_{1}$. Let $f \sim \sum c_{r} v_{r}$. Then

$$
\sum_{r} c_{r} \beta_{r k s}=\left(f * v_{k}, v_{s}\right) \text {. }
$$

Proof. The condition $a<1 / 4$ guarantees that $f * v_{k}$ will be a function of class $A$. For $f * v_{k}=F * D v_{k}$ where $F(x)=\int_{0}^{x} f$ is bounded by $C_{1} \exp \left(a x^{2}\right)$. Since $D v_{k}(x)$ is bounded by $C_{2} \exp \left(-b x^{2}\right)$ where $b=1 / 2-\epsilon$ one has

$$
\begin{aligned}
\left|\left(f * v_{k}\right)(x)\right| & \leqq C_{3} \int_{-\infty}^{\infty} \exp \left\{a(x-u)^{2}-b u^{2}\right\} d u \\
& \leqq C_{4} \exp \left\{a b x^{2} /(b-a)\right\} .
\end{aligned}
$$

Now let $R v_{k}$ be the function defined by $\left(R v_{k}\right)(x)=v_{k}(-x)$. Then a simple calculation shows that

$$
\left(f * v_{k}, v_{s}\right)=\left(f, R v_{k} * v_{s}\right) .
$$

The method of the proof preceding 56.4 shows that the series $\sum_{r} f \beta_{r k_{s}} v_{r}$ is dominatedly convergent on $(-\infty, \infty)$, and that its sum is $f\left(R v_{k} * v_{s}\right)$. Integration over $(-\infty, \infty)$ completes the proof.

57.5. Convolution $\phi * \psi$. For the ordinary convolution 41.4 one has $\left(f * g, v_{k}\right)$ $=\left(g, R \bar{f} * v_{k}\right)$ where $(R f)(x)=f(-x)$. Let $\phi \sim \sum c_{r} v_{r}, \psi \sim \sum d_{s} v_{s}$. We define $R \phi$ as $\sum c_{r} R v_{r}=\sum(-1)^{r} c_{r} v_{r}$ and then define the pansion $\phi * \psi$ by

$$
\begin{array}{rlrl}
\left(\phi * \psi, v_{k}\right) & =\left(\psi, R \bar{\phi} * v_{k}\right)=\left(\psi, \sum_{s}\left(\sum_{r}(-1)^{r} \bar{c}_{r} \beta_{r k s}\right) v_{s}\right) \\
& =\sum_{s} d_{s} \sum_{r} c_{r} \beta_{r s k}, & k=0,1, \cdots
\end{array}
$$

whenever the latter series converge.

We note the following particular cases:

$$
\begin{aligned}
(2 \pi)^{1 / 2} \delta * \psi & =\psi, \\
(2 \pi)^{1 / 2}[D] \delta * \psi & =[D] \psi .
\end{aligned}
$$

Proof. By (52.4) and 23.3 the expansion coefficients of $\delta$ and $\{1\}$ are related as follows:

$$
(2 \pi)^{1 / 2} c_{r}(\delta)=i^{r} c_{r}(1), \quad r=0,1, \cdots
$$

Hence by 57.2 and 56.4 


$$
(2 \pi)^{1 / 2} \sum_{r} c_{r}(\delta) \beta_{r s k}=i^{k}(-i)^{s} \sum_{r} c_{r}(1) \alpha_{r s k}=i^{k}(-i)^{s}\left(\eta_{k} k, v_{s}\right)=\left(v_{k}, v_{s}\right),
$$

so that by 57.5

$$
\left((2 \pi)^{1 / 2} \delta * \psi, v_{k}\right)=d_{k}=\left(\psi, v_{k}\right) .
$$

Expressing the coefficients of $[D] \delta$ in terms of those of $\delta$ one similarly has

$$
\begin{aligned}
(2 \pi)^{1 / 2} & \sum_{r}\left\{(r / 2+1 / 2)^{1 / 2} c_{r+1}(\delta)-(r / 2)^{1 / 2} c_{r-1}(\delta)\right\} \beta_{r s k} \\
= & i^{k+1}(-i)^{s} \sum_{r}\left\{(r / 2+1 / 2)^{1 / 2} c_{r+1}(1)+(r / 2)^{1 / 2} c_{r-1}(1)\right\} \alpha_{r s k} \\
= & i^{k+1}(-i)^{s}\left(\{x\} v_{k}, v_{s}\right) \\
= & \left\{\begin{array}{cl}
-(k / 2)^{1 / 2} & \text { if } s=k-1, \\
(k / 2+1 / 2)^{1 / 2} & \text { if } s=k+1, \\
0 & \text { otherwise. }
\end{array}\right.
\end{aligned}
$$

Hence by 57.5

$$
\left((2 \pi)^{1 / 2}[D] \delta * \psi, v_{k}\right)=-(k / 2)^{1 / 2} d_{k-1}+(k / 2+1 / 2)^{1 / 2} d_{k+1}=\left([D] \psi, v_{k}\right) .
$$

A simpler proof may be obtained from the theory of Fourier transforms.

VI. Fourier transforms OF PANSIONS

61. The operators $[T]$ and $[\bar{T}]$. Let $\phi$ be the pansion $\sum c_{k} v_{k}$. Then we define the Fourier transform $[T] \phi$ and the conjugate Fourier transform $[\bar{T}] \phi$ by the formulas

$$
\begin{aligned}
& {[T]_{\phi} \sim\left(\sum c_{k} T v_{k} \sim\right) \sum_{k=0}^{\infty}(-i)^{k} c_{k} v_{k}} \\
& {[\bar{T}]_{\phi} \sim\left(\sum c_{k} \bar{T} v_{k} \sim\right) \sum_{k=0}^{\infty} i^{k} c_{k} v_{k} .}
\end{aligned}
$$

If $\phi$ is a function $f$ of class $L_{1}$ then $[T] \phi=T f$ and $[\bar{T}] \phi=\bar{T} f(24)$.

The above definitions imply that

$$
\left([T]_{\phi}, v_{k}\right)=\left(\phi, \bar{T} v_{k}\right), \quad\left([\bar{T}]_{\phi}, v_{k}\right)=\left(\phi, T v_{k}\right) .
$$

61.4. InVERsion Formula. If $\psi=[T] \phi$ then $[\bar{T}] \psi=\phi$.

Proof. Let $\phi \sim \sum c_{k} v_{k}$. Then $\psi=[T] \phi \sim \sum(-i)^{k} c_{k} v_{k}$, hence $[\bar{T}] \psi$ $\sim \sum i^{k}(-i)^{k} c_{k} v_{k}=\sum c_{k} v_{k}$.

61.5. EXAMPLE. By 23.3 and 52.4

$$
[T]\{1\}=(2 \pi)^{1 / 2} \delta, \quad[T] \delta=[\bar{T}] \delta=\left\{(2 \pi)^{-1 / 2}\right\} .
$$

62. Rules for Fourier transforms. One has the following rules for $[T]$ and corresponding rules for $[\bar{T}]$ : 


$$
\begin{aligned}
{[T][D]_{\phi} } & =i[x][T]_{\phi}, \\
{[T][x]_{\phi} } & =i[D][T]_{\phi}, \\
{[T][H]_{\phi} } & =[H][T]_{\phi},
\end{aligned}
$$

and, more generally, if $E$ is an entire function,

$$
[T][E(H)]_{\phi}=[E(H)][T]_{\phi} .
$$

Also

$$
\begin{aligned}
{[T][E(D)] \phi } & =[E(i x)][T] \phi, \\
{[T][E(x)] \phi } & =[E(i D)][T]_{\phi}
\end{aligned}
$$

provided one of the two sides exists according to (54.4) or (54.3). In particular one has the following formula for the transform of a translation:

$$
[T]\left[e^{a D}\right]_{\phi}=\left[e^{i a x}\right][T]_{\phi}
$$

whenever the coefficients of $\phi$ satisfy an inequality (54.6).

Finally, for the convolution,

$$
[T](\phi * \psi)=[T]_{\phi} \cdot[T] \psi
$$

provided one of the two sides exists according to 57.5 or 56.5 .

Proofs. Let $\phi \sim \sum c_{k} v_{k}$. Then by (52.1) and (61.1)

$$
\begin{aligned}
{[T][D] \phi } & \sim \sum(-i)^{k}\left\{(k / 2+1 / 2)^{1 / 2} c_{k+1}-(k / 2)^{1 / 2} c_{k-1}\right\} v_{k} \\
& =i \sum\left\{(k / 2+1 / 2)^{1 / 2}(-i)^{k+1} c_{k+1}+(k / 2)^{1 / 2}(-i)^{k-1} c_{k-1}\right\} v_{k}
\end{aligned}
$$

which by (53.1) and (61.1) is equal to $i[x][T] \phi$. This proves (62.1); the proof of (62.2) is similar.

A proof of (62.3) and (62.4) follows from (54.1) and (54.2).

To prove (62.5) we remark that by (62.1)

$$
(-i)^{k}\left([D]^{n} \phi, v_{k}\right)=\left([T][D]^{n} \phi, v_{k}\right)=i^{n}\left([x]^{n}[T]_{\phi}, v_{k}\right) .
$$

Thus the individual terms in the sums defining

$$
\left([T][E(D)] \phi, v_{k}\right)=(-i)^{k}\left([E(D)] \phi, v_{k}\right)
$$

and $\left([E(i x)][T] \phi, v_{k}\right)$ are equal. The proof of (62.6) is similar.

To prove (62.8) we use the notation of 57.5. One has, using 57.2,

$$
\begin{aligned}
\left([T](\phi * \psi), v_{k}\right) & =(-i)^{k} \sum_{s} d_{s} \sum_{r} c_{r} \beta_{r s k} \\
& =(-i)^{k} \sum_{s} d_{s} \sum_{r} c_{r} i^{k}(-i)^{r+s} \alpha_{r s k} \\
& =\sum_{s}(-i)^{s} d_{s} \sum_{r}(-i)^{r} c_{r} \alpha_{r s k} .
\end{aligned}
$$

But by 56.5 the last sum also represents $\left([T] \phi \cdot[T] \psi, v_{k}\right)$. 
62.9. Application. Let $f$ be the Fourier transform of a function $g$ of $L_{1}$. Then

$$
\delta f=f(0) \delta .
$$

For $(2 \pi)^{1 / 2} \delta f=[T](1 * g)$. Since

$$
\left(1 * g, v_{k}\right)=\left(g, 1 * v_{k}\right)=\int_{-\infty}^{\infty} g(u) d u(2 \pi)^{-1 / 2} \int_{-\infty}^{\infty} v_{k}(x) d x
$$

the convolution $1 * g$ is equal to the constant function $\left\{(2 \pi)^{-1 / 2} \int_{-\infty}^{\infty} g(u) d u\right\}$ $=\{f(0)\}$. Hence $\delta f=(2 \pi)^{-1 / 2}[T]\{f(0)\}=f(0) \delta$.

VII. Fourier TRANSFORM THEORY FOR $L_{2}$

71. $L_{2}$ theory. The present derivation of Plancherel's theory shows some similarity with Wiener's treatment $[21$, p. 46]. Both derivations are based on Hermite expansions. However, the treatment given below is considerably shorter.

Let $f$ be a function of class $L_{2}$ and let $f$ have the Hermite expansion $\sum c_{k} v_{k}$. By the completeness of the $v_{k}$

$$
\sum_{k=0}^{\infty}\left|c_{k}\right|^{2}=(f, f)
$$

(25.1). In the spirit of this paper we define the Fourier transform of $f$ to be the Fourier transform of the pansion $\sum c_{k} v_{k}$ :

$$
[T] f=[T] \sum c_{k} v_{k}=\sum(-i)^{k} c_{k} v_{k} .
$$

That is, $[T] f$ is a pansion $\sum d_{k} v_{k}$ such that $\sum\left|d_{k}\right|^{2}$ converges. But then the Riesz-Fischer theorem asserts that there is a function $g$ in $L_{2}$ such that $g \sim \sum d_{k} v_{k}$ (25.1). By our convention we identify $\sum d_{k} v_{k}$ with $g$. Note that

$$
(g, g)=\sum\left|d_{k}\right|^{2}=\sum\left|c_{k}\right|^{2}=(f, f) \text {. }
$$

Application of the inversion formula 61.4 completes the proof of the basic theorem.

71.2. THEOREM. Let $f$ be a function of $L_{2}$. Then $g=[T] f$ is also a function of $L_{2}$. One has $[\bar{T}] g=f$ and

$$
([T] f,[T] f)=(f, f) .
$$

72. Plancherel's definition. Plancherel was the first to prove that the class $L_{2}$ is closed under Fourier transformation. He used a different definition of the Fourier transform of a function of $L_{2}$. However, we will show that our definition leads to the same Fourier transform as his original definition.

72.1. Plancherel's theorem. Let $f$ be in $L_{2}$. Then the functions 


$$
h_{a}(x)=(2 \pi)^{-1 / 2} \int_{-a}^{a} f(u) e^{-i x u} d u
$$

converge in the mean on $(-\infty, \infty)$ to a function $h$ of $L_{2}$ as $a \rightarrow \infty$. One has $(h, h)$ $=(f, f)$. Reciprocally the functions

$$
k_{a}(x)=(2 \pi)^{-1 / 2} \int_{-a}^{a} h(u) e^{i x u} d u
$$

converge in the mean to $f$ as $a \rightarrow \infty$.

Proof. We will show that the functions $h_{a}(x)$ converge in the mean to $g=[T] f$.

Let $f_{a}(x)$ be equal to $f(x)$ for $|x| \leqq a$ and equal to 0 for $|x|>a$. Then $f_{a}$ is in $L_{1}$ (Schwarz's inequality) and hence $h_{a}=T f_{a}=[T] f_{a}(61)$. By 71.2 the transform $g=[T] f$ is in $L_{2}$. Clearly

$$
g-h_{a}=[T]\left(f-f_{a}\right) .
$$

The function $f-f_{a}$ is in $L_{2}$. Hence by 71.2

$$
\int_{-\infty}^{\infty}\left|g-h_{a}\right|^{2} d x=\int_{-\infty}^{\infty}\left|f-f_{a}\right|^{2} d x=\left(\int_{-\infty}^{-a}+\int_{a}^{\infty}\right)|f|^{2} d x .
$$

As $a \rightarrow \infty$ the right hand side approaches 0 , hence $h_{a}$ converges in the mean to g. This proves that Plancherel's Fourier transform $h$ of $f$ exists and that $h=g$. The reciprocal relation may now be proved in the same way.

VIII. FOURIER TRANSFORM THEORY FOR PANSIONS OF POLYNOMIAL GROWTH

81. Pansions of polynomial growth. The example $[T]\{1\}=(2 \pi)^{1 / 2} \delta(61.5)$ shows that the transform of a function of polynomial growth need not be a function of polynomial growth (52.6).

By 32.3 every function $f$ of at most polynomial growth may be written in the form $H^{n} f_{0}$ where $f_{0} \in L_{2}$. Or, using a weaker formulation (54.1) which could actually be derived in a simpler fashion,

$$
f=[H]^{n} f_{0}, \quad f_{0} \in L_{2} .
$$

Hence since $[T]$ and $[H]$ commute (62.3)

$$
[T] f=[H]^{n} g_{0}, \quad g_{0}=[T] f_{0} \in L_{2} .
$$

In order to obtain a class of objects which contains all functions of polynomial growth and which is closed under Fourier transformation we are thus led to the following:

81.1. Definition. $A$ pansion $\phi$ is said to be of polynomial growth if it can be written in the form

$$
\phi=[H]^{n} f_{1},
$$$$
f_{1} \in L_{2}
$$ 
where $n$ is an integer $\geqq 0$.

81.3. Theorem. The class of pansions of polynomial growth is closed under Fourier transformation (as well as conjugate Fourier transformation).

Proof. Let $\phi$ be of the form (81.2). Then

$$
[T]_{\phi}=[H]^{n}[T] f_{1}=[H]^{n} g_{1}, \quad g_{1} \in L_{2} .
$$

In the next section we will give several characterizations of the class of pansions of polynomial growth. We will show in particular that a pansion is of polynomial growth if and only if it is the global derivative of some finite order of a function of at most polynomial growth. Thus the pansions of polynomial growth correspond to Laurent Schwartz's tempered distributions, that is, the class of distributions for which Schwartz defines a Fourier transform [16, II, p. 79].

82. Characterizations of pansions of polynomial growth. We will prove

82.1. Characterization theorem. The following four statements about a pansion $\phi \sim \sum c_{k} v_{k}$ are equivalent:

(i) $\phi$ is a pansion of polynomial growth:

$$
\phi=[H]^{n} f_{1},
$$

(ii) $\phi$ can be written as a finite order global derivative of a function of at most polynomial growth:

$$
\phi=[D]^{p}\left(x^{2}+1\right)^{q} f_{2}, \quad f_{2} \text { in } L_{2} \text { and bounded, }
$$

(iii) $\phi$ can be written as the global product of a power of [x] and a global polynomial derivative of a function of $L_{2}$ :

$$
\phi=[x]^{r}\left[D^{2}-1\right]^{s} f_{3}, \quad f_{3} \in L_{2} .
$$

(iv) there are constants $M$ and $m$ such that

$$
\left|c_{k}\right| \leqq M k^{m}, \quad k=1,2, \cdots \text {. }
$$

Proof. (i) $\rightarrow$ (ii). Let (i) hold. In the case $n=0$ one has $f_{1}=[D] f$ where $f(x)=\int_{0}^{x} f_{1}$ is a function of at most polynomial growth.

Now let $n \geqq 1$ and suppose it has been shown already that $\psi=[H]^{n-1} f_{1}$ is equal to $[D]^{2 n-1} g$ where $g$ is a function of polynomial growth. One has $\phi=[H] \psi=[x]^{2} \psi-[D]^{2} \psi$. Hence it is sufficient to show that $[x] \chi$ is equal to a global derivative of order $2 n-1$ of a function of polynomial growth whenever $\chi$ is. Set $\chi=[D]^{2 n-1} h$. Then by 53.4

$$
[x] \chi=[x][D]^{2 n-1} h=[D]^{2 n-1}\left(\{x\} h-(2 n-1) \int_{0}^{x} h\right)
$$

and the result follows.

The above proof shows more precisely that (i) implies the representation 


$$
\phi=[D]^{2 n+1} f
$$

where $|f(x)| \leqq C(|x|+1)^{4 n+1 / 2}$.

(i) $\rightarrow$ (iii). Let (i) hold. Then $\omega=[\bar{T}] \phi$ is also a pansion of polynomial growth (81.3), hence we may apply (ii) to obtain

$$
\omega=[D]^{r}\left(x^{2}+1\right)^{8} f_{4}, \quad f_{4} \in L_{2} .
$$

By the inversion formula 61.4 and other rules for Fourier transforms (62.1), (62.6)

$$
\begin{aligned}
\phi & =[T] \omega=i^{r}[x] r\left[i^{2} D^{2}+1\right] s[T] f_{4} \\
& =[x]^{r}\left[D^{2}-1\right]^{s} f_{3}
\end{aligned}
$$

where $f_{3}=i^{r+2 s}[T] f_{4} \in L_{2}$.

(ii) $\rightarrow$ (iv) and (iii) $\rightarrow$ (iv). It will be sufficient to prove the first of these. Let (ii) hold. The expansion coefficients of $f_{2} \in L_{2}$ are bounded (25.1). Hence the coefficients $d_{k}$ of $\left(x^{2}+1\right)^{\alpha} f_{2}$ are bounded by a constant times $(k+1)^{q}$ (23.5), and it follows from the definition of $[D]$ (52.1) that the coefficients $c_{k}$ of $\phi$ are bounded by a constant times $(k+1)^{q+p / 2}$.

(iv) $\rightarrow$ (i). Let (iv) hold. Let $n$ be an integer $\geqq 0$ and $>m+1 / 2$. Then the numbers $e_{k}=(2 k+1)^{-n} c_{k}$ are the expansion coefficients of a function $f_{1} \in L_{2}$ since $\sum\left|e_{k}\right|^{2}$ converges (25.1). And by the definition of $[H]$ (54.1) we have $\phi=[H]^{n} f_{1}$.

83. Applications. We can now prove the following result in addition to 81.3.

83.1. Theorem. The class of pansions of polynomial growth is closed under the operations listed: $[H]$, global differentiation $[D]$, global multiplication by $[x]$, translation $\left[e^{a D}\right]$, global multiplication by $\left[e^{i b x}\right]$ (provided $a$ and $b$ are real).

Proof. The statement on $[H],[D]$ and $[x]$ follows from 82.1 .

Once more using the representation $\phi=[D]^{p} f, f$ a function of polynomial growth, one has (54.7)

$$
\left[e^{a D}\right]_{\phi}=\left[e^{a D}\right][D]^{p} f=[D]^{p}\left[e^{a D}\right] f
$$

and by 55.1, $\left[e^{a_{D}}\right] f=\{f(x+a)\}$. Thus $\left[e^{a_{D}}\right] \phi$ is a pansion of polynomial growth.

Finally by (55.6)

$$
\left[e^{i b x}\right]_{\phi}=\left[e^{i b x}\right][D]^{p} f=[D-i b]^{p}\left\{e^{i b x}\right\} f
$$

which shows that $\left[e^{i b x}\right] \phi$ is a pansion of polynomial growth.

83.2. COROLlaRy. The class of pansions of polynomial growth may be characterized as the smallest vector space which contains $L_{2}$ and which is closed under global differentiation $[D]$ and global multiplication by $[x]$. 
For every vector space with the properties stated contains all $\phi$ 's of the form 82.1 (ii).

Alternate computation of Fourier transforms. In the case of a function $f$ of at most polynomial growth the present Fourier transform $[T] f$ is essentially equal to the global $k$ th derivative of Bochner's " $k$-Transformierte" of $f[3$, p. 110]:

83.3. Theorem. Let $f$ be a function such that $f /\{|x|+1\}^{k}$ is in $L_{1}, k$ an integer $\geqq 0$. Define $f_{1}(x)=f(x)$ for $|x| \leqq 1, f_{1}(x)=0$ for $|x|>1$, and set $f_{2}=f-f_{1}$. Then

$$
[T] f=T f_{1}+[D]^{k} T\left(f_{2} /\{-i x\}^{k}\right)
$$

where $T$ is the ordinary $L_{1}$ Fourier transformation.

Proof. Since $f_{1}$ and $f_{3}=f_{2} /\{x\}^{k}$ are in $L_{1}$ one has

$$
[T] f_{1}=T f_{1}, \quad[T] f_{3}=T f_{3}
$$

(61). Hence by rule (62.2)

$$
[T] f_{2}=[T]\left(\{x\}^{k} f_{3}\right)=[i D]^{k} T f_{3} .
$$

We may apply the preceding result to obtain a formula for the Fourier transform of any pansion of polynomial growth:

83.4. TheOREM. Let $\phi$ be a pansion of polynomial growth. Then we may write $\phi=[D]^{p} f$ where $p \geqq 0$ and $f /\{|x|+1\}^{k} \in L_{1}$ for some integer $k \geqq 0$. Using the notation of 83.3 one has

$$
[T]_{\phi}=\{i x\}^{p} T f_{1}+[i x]^{p}[i D]^{k} T\left(f_{2} /\{x\}^{k}\right) .
$$

The proof follows from 82.1 which gives the representation $\phi=[D]^{p} f$, 83.3 and (62.1).

83.4 shows once more that $[T] \phi$ is a pansion of polynomial growth.

IX. FouRIER TRANSFORM THEORY FOR PANSIONS OF EXPONENTIAL GROWTH

91. Pansions of exponential growth. By 35.1 every function $f$ of at most exponential growth may be written in the form $E(H) f_{0}$ where $f_{0} \in L_{2}$ and $E\left(z^{2}\right)$ is an even entire function of $z$ of exponential type. More precisely, if $f$ is of exponential type $m$ then $E\left(z^{2}\right)$ may be taken of exponential type $m$.

Using a weaker formulation which could actually be derived in a much simpler fashion we have the representation (54.2)

$$
f=[E(H)] f_{0},
$$$$
f_{0} \in L_{2}
$$

Hence since $[T]$ and $[E(H)]$ commute (62.4)

$$
[T] f=[E(H)] g_{0}, \quad g_{0}=[T] f_{0} \in L_{2} .
$$

In order to obtain a class of objects which contains all functions of ex- 
ponential growth and which is closed under Fourier transformation we are thus led to the following:

91.1. Definition. $A$ pansion $\phi$ is said to be of exponential growth if it can be written in the form

$$
\phi=[E(H)] f_{1}, \quad f_{1} \in L_{2},
$$

where $E$ is an entire function such that $E\left(z^{2}\right)$ is of exponential type as a function of $z$.

A pansion $\phi$ is said to be of exponential type $m$ if it can be written in the form (91.2) with $E\left(z^{2}\right)$ of type $m$, but not with $E\left(z^{2}\right)$ of type $<m$. A function $f$ of exponential type $m$ will be of type $\leqq m$ when considered as a pansion.

91.3. ThEOREM. The class of pansions of exponential growth is closed under Fourier transformation.

For if $\phi$ has the form (91.2) then

$$
[T]_{\phi}=[E(H)][T] f_{1}=[E(H)] g_{1},
$$

In the next sections we will obtain several characterizations of the class of pansions of exponential growth. It will be shown in particular that $a$ pansion is of exponential growth if and only if it is an infinite order global derivative of exponential type of a function of at most exponential growth.

92. Characterization by coefficients. We will prove the following:

92.1. Characterization theorem. A pansion $\phi \sim \sum c_{k} v_{k}$ is of exponential growth if and only if there are constants $M$ and $b$ such that

$$
\left|c_{k}\right| \leqq M \exp \left\{b(2 k)^{1 / 2}\right\}, \quad k=0,1, \cdots .
$$

The type of $\phi$ as defined in 91 is equal to

$$
l=\max (\lambda, 0), \quad \lambda=\limsup _{k \rightarrow \infty} \frac{\log \left|c_{k}\right|}{(2 k)^{1 / 2}} .
$$

Proof. (i) Let $\phi$ be of the form (91.2) with $E\left(z^{2}\right)$ an even entire function of $z$ of exponential type $m$. The coefficients $d_{k}$ of $f_{1} \in L_{2}$ are bounded (25.1). Hence the coefficients $c_{k}=E(2 k+1) d_{k}$ of $\phi(54.2)$ are bounded by an expression of the form $M(\epsilon) \exp \left\{(m+\epsilon)(2 k+1)^{1 / 2}\right\}$ for every $\epsilon>0$. This proves (92.2). It also follows that the number $l$ in (92.3) has a value $\leqq m$. In particular $l$ can not exceed the type of $\phi$.

(ii) Let $\phi$ be a pansion whose coefficients $c_{k}$ satisfy (92.2). Then $l=\max$ $(\lambda, 0)$ is finite, and the function $f$ defined by

$$
f(x)=\left|c_{k}\right|, \quad(2 k)^{1 / 2} \leqq|x|<(2 k+2)^{1 / 2}, \quad k=0,1, \cdots
$$

will be of type $l$ on the real axis. Thus by 33.3 there exists an entire function $E_{0}(z)$ such that $E_{0}\left(z^{2}\right)$ is of exponential type $l$ as a function of $z$ while $f(x)$ $\leqq E_{0}\left(x^{2}\right)$. That is, $\left|c_{k}\right| \leqq E_{0}(2 k+1), k=0,1, \cdots$. 
Now define

$$
d_{k}=c_{k} /(2 k+1) E_{0}(2 k+1), \quad k=0,1, \cdots,
$$

and let $f_{1}$ be the function of $L_{2}$ which has the numbers $d_{k}$ as its expansion coefficients (25.1). Then

$$
\phi=\left[H \cdot E_{0}(H)\right] f_{1} .
$$

It follows that $\phi$ is a pansion of exponential growth. Moreover the type of $\phi$ does not exceed the type $l$ of $z^{2} E_{0}\left(z^{2}\right)$.

We can now prove the following result in addition to 91.3.

92.4. THEOREM. The class of pansions of exponential growth is closed under the following operations: the taking of infinite order global derivatives $[E(D)]$ and global multiplication by $[E(x)]$ where $E$ is any entire function of exponential type. If $\phi$ is of type $m$ and $E$ of type $p$ then $[E(D)] \phi$ and $[E(x)] \phi$ are of type $\leqq m+p$.

Proof. Let $\phi \sim \sum c_{k} v_{k}$ be of type $m$. By 92.1 we will have an inequality (92.2) for every $b>m$. Let $E$ be of type $p$. Then $E(x)=\sum a_{n} x^{n}$ where the $a_{n}$ satisfy an inequality $\left|a_{n}\right| \leqq R r^{n} / n$ ! for every $r>p[2$, p. 11].

Just as in 25.2

$$
\begin{aligned}
\left|\left([x]^{n} \phi, v_{k}\right)\right| & \leqq\{(k+n) \cdots(k+1)\}^{1 / 2} 2^{-n / 2}\left(\left|c_{k+n}\right|+C_{n, 1}\left|c_{k+n-2}\right|+\cdots\right) \\
& \leqq\{(k+n) \cdots(k+1)\}^{1 / 2} 2^{n / 2} M \exp \left\{b(2 k+2 n)^{1 / 2}\right\} .
\end{aligned}
$$

Hence by (54.3) and the inequality for the $a_{n}$,

$$
\begin{aligned}
& \left|\left([E(x)] \phi, v_{k}\right)\right|=\left|\sum_{n} a_{n}\left([x]^{n} \phi, v_{k}\right)\right| \\
& \quad \leqq M R \sum_{n}\{(k+n) \cdots(k+1)\}^{1 / 2} \exp \left\{b(2 k+2 n)^{1 / 2}\right\} \cdot\left(2^{1 / 2} r\right)^{n} / n ! .
\end{aligned}
$$

Take $k$ large. Then the maximum term in the above series has an index $n$ roughly equal to $(2 k)^{1 / 2} r$. Comparing the sum of the series with the maximum term one finds by routine calculation that

$$
\left|\left([E(x)]_{\phi}, v_{k}\right)\right| \leqq S(\epsilon) \exp \left\{(b+r+\epsilon)(2 k)^{1 / 2}\right\},
$$

where $\epsilon>0$ is arbitrary. Since we may take $b=m+\epsilon$ and $r=p+\epsilon$ it follows that $[E(x)] \phi$ is of type $\leqq m+p$.

The proof for $[E(D)] \phi$ is similar.

93. The basic representation theorem. Instead of a pansion we first consider a function $g$, of class $L_{1}$, say. We try to write $g$ in the form

$$
g=[\cos a D] f,
$$$$
a>0,
$$

where $f$ is another function of $L_{1}$. Taking Fourier transforms we obtain (62.5)

$$
T g=[\cosh a x] T f .
$$


One solution of this equation is

$$
T f=\{1 / \cosh a x\} T g ;
$$

the general solution differs from it by an infinite linear combination of translates of $\delta$ (55.5), (62.9).

An easy calculation shows that $\{1 / \cosh a x\}$ is the Fourier transform of $\left\{(\pi / 2)^{1 / 2} / a \cosh (\pi x / 2 a)\right\}$. Hence the Convolution Theorem 41.4 leads one to expect that

$$
f(x)=(1 / 2 a) \int_{-\infty}^{\infty} \frac{1}{\cosh \{\pi(x-u) / 2 a\}} g(u) d u
$$

will be a particular solution of (93.1). This result is actually true even if $g$ is a function of exponential growth, provided the type of $g$ is less than $\pi / 2 a$. A proof may be given by computation of the Hermite expansion of $[\cos a D] f$ where $f$ is given by (93.2) (see 95). Or one may use the methods of Hirschman and Widder [9, p. 16].

Now consider the equation

$$
\phi=[\cos a D] f,
$$

$a>0$,

where $\phi$ is a given pansion of exponential growth. Then $\phi=[E(H)] f_{1}$ where $f_{1} \in L_{2}, E\left(z^{2}\right)$ of exponential type. Proceeding as above one obtains a formal solution of (93.3) in the form (93.2) with $g$ replaced by $\phi=[E(H)] f_{1}$. To find the proper interpretation of this answer we recall that $H$ is self-adjoint (14.1). It follows that $E(H)$ is self-adjoint, hence one is led to the conjecture that a particular solution of (93.3) is given by

(93.4) $f(x)=(1 / 2 a) \int_{-\infty}^{\infty} f_{1}(u) E(H) \frac{1}{\cosh \{\pi(x-u) / 2 a\}} d u, \quad H=u^{2}-d^{2} / d u^{2}$.

Unfortunately there is a complication which makes the solution (93.4) valid only when $E\left(z^{2}\right)$ is of sufficiently small exponential type. The reason for this restriction is as follows. $E(H)(1 / \cosh b u)$ will exist for small $u$ only when $1 / \cosh b u$ is analytic in a sufficiently large disc about the origin. That means that $b$ must be small. On the other hand $E(H)(1 / \cosh b u)$ will go to 0 as $u \rightarrow \infty$ only when $b$ is sufficiently large. No value of $b$ will satisfy both requirements unless $E\left(z^{2}\right)$ is of small type.

One can get around this difficulty by replacing equation (93.3) by the equation

$$
\phi=\left[\cos a D / \prod_{j \leqq 8}\left(1-D^{2} / p_{j}^{2}\right)\right] f^{*},
$$$$
a>0,
$$

where $p_{j}=(j-1 / 2) \pi / a$. Proceeding as before we now need the conjugate Fourier transform of 


$$
\left\{(1 / \cosh a x) \cdot \prod_{j \leq 8}\left(1+x^{2} / p_{j}^{2}\right)\right\} .
$$

Induction with respect to $s$ shows that the above function is the Fourier transform of

$$
(\pi / 2)^{1 / 2}\{2 \cdot 4 \cdots 2 s / 1 \cdot 3 \cdots(2 s-1) a\} \cdot\{\cosh (\pi x / 2 a)\}^{-2 z^{-1}} .
$$

Hence one may expect that for suitable $a$ and $s$ equation (93.5) will have the particular solution

$$
\begin{array}{r}
f^{*}(x)=\frac{2}{1} \cdots \frac{2 s}{2 s-1} \cdot \frac{1}{2 a} \int_{-\infty}^{\infty} f_{1}(u) E(H) \frac{1}{[\cosh \{\pi(x-u) / 2 a\}]^{2 s+1}} d u, \\
H=u^{2}-d^{2} / d u^{2}
\end{array}
$$

In order to obtain a solution $f$ of (93.3) one finally has to solve the ordinary differential equation

$$
f^{*}=\prod_{j \leqq 8}\left(1-D^{2} / p_{j}^{2}\right) f .
$$

After some further preparations in 94 we will in 95 prove the following:

93.8. REPRESENTATION THEOREM. Let $\phi$ be a pansion of exponential type $m$. That is, we may write $\phi=[E(H)] f_{1}$ where $f_{1} \in L_{2}$ and $E$ is an entire function such that $E\left(z^{2}\right)$ is of exponential type $m$ as a function of $z$. Then for $a>18 m^{2}$ $+2 \log 2$ and $s \geqq 0$ so large that $\pi(s+1 / 2)>3 m a$ one has the representation (93.3) where $f$ is a function of exponential growth given by (93.7) and (93.6).

94. Lemmas on $E(H)$. We will prove a few lemmas which will be used in the proof of 93.8 .

Throughout this section we will assume that $E\left(z^{2}\right)$ is of exponential type $m$. Then $E(z)=\sum a_{n} z^{n}$ where the $a_{n}$ satisfy a set of inequalities

$$
\left|a_{n}\right| \leqq M \mu^{2 n} /(2 n) !, \quad n=0,1, \ldots
$$

for every $\mu>m[2$, p. 11$]$.

The letter $H$ will stand for $u^{2}-d^{2} / d u^{2}$.

94.2. Lemma. Let $F(x)=\sum d_{p} x^{p}$ where for some $\rho>8 m$

$$
\left|d_{p}\right| \leqq R^{-p}, \quad \quad p=0,1, \cdots
$$

Then the series $\sum a_{n} H^{n} F(x-u)$ is convergent for. $|x-u|<\rho / 2$. One has the following inequalities for the partial sums and the sum of the series:

$$
\left.\mid \begin{array}{l}
\left|\sum_{n=0}^{N} a_{n} H^{n} F(x-u)\right| \\
|E(H) F(x-u)|
\end{array}\right\} \leqq M_{1} \exp \left(M_{2}|x|\right), \quad|x-u| \leqq \rho / 2-\epsilon,
$$


where the $M_{i}$ are independent of $x$ and $u$.

Proof. One has first of all

$$
H^{n}(u-x)^{p}=\sum_{\max } A_{(-2 n,-p) \leq j \leq 2 n} A_{n j}(u-x)^{p+j}, \quad p \geqq 0, n \geqq 0,
$$

where

$$
A_{n+1, j}=A_{n, j-2}+2 x A_{n, j-1}+x^{2} A_{n j}-(p+j+2)(p+j+1) A_{n, j+2} .
$$

Hence by induction with respect to $n$

$$
\begin{aligned}
\left|A_{n j}\right| & \leqq 3^{n}(1+\xi)^{n+j / 2}(p+2 n+\xi) ! /\{(p+\xi) !(n+j / 2) !\} \\
& \leqq 3^{n} 2^{2 n+p+\xi}(1+\xi)^{n+j / 2}(2 n) ! /(n+j / 2) !
\end{aligned}
$$

where $\xi=|x|+2$.

We next consider the series

$$
H^{n} F(x-u)=\sum_{p=0}^{\infty} d_{p} H^{n}(x-u)^{p}
$$

where we take $|x-u| \leqq \rho / 2-\epsilon$. By (94.3) the sum is bounded by

$$
\begin{aligned}
& \left|H^{n} F(x-u)\right| \\
& \leqq R 3^{n} 2^{2 n+\xi}(2 n) ! \sum_{j=-2 n}^{2 n}(1+\xi)^{n+j / 2} /(n+j / 2) ! \sum_{p \geq \max (-j, 0)} 2^{p}|x-u|^{p+i} / \rho^{p}
\end{aligned}
$$

$$
\begin{aligned}
= & R 3^{n} 2^{2 n+\xi}(2 n) !\left\{\sum_{j=-2 n}^{0} \sum_{p \geqq-j}+\sum_{j=1}^{2 n} \sum_{p \geqq 0}\right\} \\
\leqq & M_{3} 3^{n} 2^{2 n+\xi}(2 n) !\left[(\rho / 2)^{-2 n} \exp \left\{\rho^{2}(1+\xi) / 4\right\}\right. \\
& \left.+2 n(1+\xi)^{n} / n !+2 n(1+\xi)^{2 n}|x-u|^{2 n} /(2 n) !\right] .
\end{aligned}
$$

We finally turn to the series $\sum a_{n} H^{n} F(x-u)$. Estimate (94.4) combined with (94.1) with $m<\mu<\rho / 8$ implies that this series converges uniformly for $|x-u| \leqq \rho / 2-\epsilon,-c \leqq x \leqq c$. It also follows that for $|x-u| \leqq \rho / 2-\epsilon$ the partial sums and the sum $E(H) F(x-u)$ are bounded by the expression given in 94.2.

94.5. Lemma. Let $F(x)=\sum e_{p} \exp \{(\alpha+p \beta) x\}$ where $\alpha>0, \beta>0$ and

$$
\left|e_{p}\right| \leqq S e^{p \sigma},
$$$$
p=0,1, \cdots \text {. }
$$

Then the series $\sum a_{n} H^{n} F(x-u)$ is convergent for $u-x>9 m^{2}+\log 2+\sigma / \beta$. One has the following inequalities for the partial sums and the sum of the series:

$$
\left.\begin{array}{c}
\left|\sum_{n=0}^{N} a_{n} H^{n} F(x-u)\right| \\
|E(H) F(x-u)|
\end{array}\right\} \leqq M_{4} \exp \{\alpha(x-u)+(3 m+\epsilon)|u|\},
$$


provided $u-x \geqq 9 m^{2}+\log 2+\sigma / \beta+\epsilon$. Here $M_{4}$ is independent of $x$ and $u$.

Proof. One has for real $t$

where

$$
H^{n} e^{t u}=\sum_{j=0}^{2 n} B_{n j} u^{j} e^{t u}
$$

$$
B_{n+1, j}=B_{n, j-2}-(j+2)(j+1) B_{n, j+2}-2 t(j+1) B_{n, j+1}-t^{2} B_{n j} .
$$

Hence by induction with respect to $n$

$$
\begin{aligned}
\left|B_{n j}\right| & \leqq 2^{n}(1+\tau)^{n-j / 2}(2 n+\tau) ! /\{\tau ! j !(n-j / 2) !\} \\
& \leqq 2^{3 n+\tau}(1+\tau)^{n-j / 2}(2 n) ! /\{j !(n-j / 2) !\}
\end{aligned}
$$

where $\tau=|t|$.

Combining (94.1) and (94.6) we obtain the estimate

$$
\begin{aligned}
& \left|\sum_{n=0}^{N} a_{n} H^{n} e^{t u}\right| \leqq \sum_{n=0}^{\infty}\left|a_{n}\right|\left|H^{n} e^{t u}\right| \\
& \quad \leqq M 2^{\tau} e^{t u} \sum_{j=0}^{\infty}|u| j / j ! \sum_{n \geq j / 2}(3 \mu)^{2 n}(1+\tau)^{n-j / 2} /(n-j / 2) ! \\
& \quad \leqq M_{5} 2^{\tau} e^{t u} \exp \left\{9 \mu^{2}(1+\tau)\right\} \sum_{j=0}^{\infty}\{3 \mu|u|\}^{j} / j ! \\
& \quad=M_{6} \exp \left\{\left(9 \mu^{2}+\log 2\right)|t|\right\} \exp \{t u+3 \mu|u|\} .
\end{aligned}
$$

We now turn to the series $\sum a_{n} H^{n} F(x-u)$. Estimate (94.7) implies that

$$
\begin{aligned}
& \sum_{n=0}^{\infty}\left|a_{n}\right|\left|H^{n} F(x-u)\right| \leqq \sum_{p=0}^{\infty} S e^{p \sigma} \sum_{n=0}^{\infty}\left|a_{n}\right|\left|H^{n} \exp \{(\alpha+p \beta)(x-u)\}\right| \\
& \leqq M_{7} \exp \{\alpha(x-u)+3 \mu|u|\} \sum_{p=0}^{\infty} \exp \left\{p \beta\left(9 \mu^{2}+\log 2+\sigma / \beta+x-u\right)\right\} .
\end{aligned}
$$

The result follows since $\mu$ may be any number $>m$.

95. Proof of Representation Theorem 93.8. Let

$$
F(x)=(\cosh b x)^{-2 s-1},
$$$$
b=\pi / 2 a, s \geqq 0,
$$

and let $E\left(z^{2}\right)=\sum a_{n} z^{2 n}$ be of exponential type $m$. By 94.2 and 94.5 with $\rho=a-\epsilon, \sigma=\epsilon$ the partial sums of the series $\sum a_{n} H^{n} F(x-u)$ as well as its sum $E(H) F(x-u)$ are bounded by

$M_{1} \exp \left(M_{2}|x|\right)$ for $|x-u| \leqq a / 2-\epsilon$, $M_{4} \exp \{\pi(s+1 / 2)(x-u) / a+(3 m+\epsilon)|u|\}$ for $u-x \geqq 9 m^{2}+\log 2+\epsilon$, provided $a>8 \mathrm{~m}$. An inequality similar to the second one holds for $u-x$ $\leqq-9 m^{2}-\log 2-\epsilon$. 
Now choose

$$
a>18 m^{2}+2 \log 2, \quad \pi(s+1 / 2)>3 m a .
$$

(Hence $a>8 m$.) Then the above inequalities cover all values of $x$ and $u$, and we can summarize our information as follows.

95.3. Lemma. Let $F(x)$ be given by (95.1) where a and satisfy (95.2). Let $E\left(z^{2}\right)=\sum a_{n} z^{2 n}$ be of exponential type $m$. Then there are positive constants $c_{i}$ such that

$$
\left.\begin{array}{c}
\left|\sum_{n=0}^{N} a_{n} H^{n} F(x-u)\right| \\
|E(H) F(x-u)|
\end{array}\right\} \leqq c_{1} \exp \left(c_{2}|x|-c_{3}|u|\right),
$$

$-\infty<x, u<\infty, N=0,1, \cdots$.

Proof of 93.8. Let $\phi=[E(H)] f_{1}$ where $f_{1} \in L_{2}$ and $E\left(z^{2}\right)$ is of exponential type $m$. Let $a$ and $s$ satisfy (95.2) and define $f^{*}$ by (93.6). By 95.3 the function $f^{*}$ will be of exponential growth. Finally let $f$ be any solution of (93.7). Then $f$ will also be of exponential growth. We have to show that $\phi=[\cos a D] f$.

We will use the notation

(95.4) $G(D)=\cos a D / \prod_{j \leqq 8}\left(1-D^{2} / p_{j}^{2}\right)=\sum \alpha_{2 \nu} D^{2 \nu}, \quad p_{j}=(j-1 / 2) \pi / a$.

Then by (93.7) (compare 54.7)

$$
[\cos a D] f=[G(D)] f^{*} .
$$

By (54.4) and (52.3)

$$
\begin{aligned}
\left([G(D)] f^{*}, v_{k}\right) & =\sum \alpha_{2 v}\left([D]^{2 v} f^{*}, v_{k}\right) \\
& =\sum \alpha_{2 v}\left(f^{*}, D^{2 v} v_{k}\right)=\left(f^{*}, G(D) v_{k}\right) .
\end{aligned}
$$

The last step may be justified by dominated convergence: we have the inequalities

$$
\left.\begin{array}{c}
\left|\sum_{\nu=0}^{N} \alpha_{2 \nu} D^{2 v} v_{k}(x)\right| \\
\left|G(D) v_{k}(x)\right|
\end{array}\right\} \leqq C(|x|+1)^{k} \exp \left(|2 a x|-x^{2} / 2\right),
$$

$-\infty<x<\infty, N=0,1, \cdots$ (compare 55).

We now replace $f^{*}$ in the last scalar product of (95.6) by its definition (93.6). In the resulting repeated integral we may invert the order of integration because of 95.3, (95.7) and Fubini's theorem. We obtain

$$
\left(f^{*}, G(D) v_{k}\right)=\gamma \int_{-\infty}^{\infty} f_{1}(u) d u \int_{-\infty}^{\infty} E(H) F(x-u) \cdot G(D) v_{k}(x) d x
$$


where $F$ is given by $(95.1), \gamma=2 \cdot 4 \cdots(2 s) /\{1 \cdot 3 \cdots(2 s-1) 2 a\}$.

We will first consider the integral with respect to $x$ in (95.8). By 95.3 and (95.7) the series

$$
\sum a_{n} H^{n} F(x-u) \cdot G(D) v_{k}(x) \quad(u, k \text { fixed })
$$

is dominatedly convergent on $-\infty<x<\infty$. Hence it may be integrated term by term and it follows that the integral with respect to $x$ in (95.8) is equal to

$$
E(H) \int_{-\infty}^{\infty} F(x-u) G(D) v_{k}(x) d x .
$$

In order to compute the integral in (95.9) we remark that $F$ and $G(D) v_{k}$ have the following Fourier transforms (compare 93 and (62.5)):

$$
(2 \pi)^{-1 / 2}(1 / \gamma)\left\{(1 / \cosh a x) \prod_{j \leqq s}\left(1+x^{2} / p_{j}^{2}\right)\right\}, \quad\left\{G(i x)(-i)^{k} v_{k}(x)\right\} .
$$

Hence by the Convolution Theorem 41.4 and the definition of $G$ (95.4) the Fourier transform of the integral in (95.9) equals $(1 / \gamma)(-i)^{k} v_{k}$. Thus by the Inversion Theorem 41.3 the integral in $(95.9)$ is equal to $(1 / \gamma) v_{k}(u)$.

Collecting results, starting with (95.5),

$$
\left([\cos a D] f, v_{k}\right)=\left(f^{*}, G(D) v_{k}\right)=\left(f_{1}, E(H) v_{k}\right),
$$

which by the definition of $[E(H)](54.2)$ equals

$$
\left([E(H)] f_{1}, v_{k}\right)=\left(\phi, v_{k}\right) .
$$

That is, the pansions $[\cos a D] f$ and $\phi$ have the same coefficients, hence they are identical.

96. Applications. We will first prove the following

96.1. Basic characterization theorem. A pansion is of exponential growth if and only if it can be written as an infinite order global derivative of exponential type of a function of at most exponential growth.

The proof follows immediately from 93.8 and 92.4 . We can also say that the class of pansions of exponential growth is the class of pansions of the form (96.2)

$$
\phi=[\cos a D]\{\cosh b x\} f_{2}, \quad a \geqq 0, b \geqq 0, f_{2} \in L_{2} .
$$

96.3. Corollary. The class of pansions of exponential growth may be characterized as the smallest class of pansions which contains $L_{2}$ and which is closed under the operations $[E(D)]$ and $[E(x)]$ for every entire function $E$ of exponential type.

Alternate computation of Fourier transforms. Let $f$ be a given function. In what follows we shall define $f_{+}(x)=f(x)$ for $x \geqq 0, f_{+}(x)=0$ for $x<0$, and $f_{-}=f-f_{+}$. 
96.4. ThEOREM. Let $f$ be a function such that $\left\{e^{-m x}\right\} f_{+}$and $\left\{e^{p x}\right\} f_{-}$are in $L_{1}$. Then

$$
[T] f=\left[e^{i m D}\right] T\left\{e^{-m x}\right\} f_{+}+\left[e^{-i p D}\right] T\left\{e^{p x}\right\} f_{-}
$$

where $T$ is the ordinary $L_{1}$ Fourier transformation.

Proof. One has $[T] f=[T] f_{+}+[T] f_{-}$. Furthermore

$$
[T] f_{+}=[T]\left\{e^{m x}\right\}\left\{e^{-m x}\right\} f_{+}=\left[e^{i m D}\right] T\left\{e^{-m x}\right\} f_{+}
$$

by (62.6) and because $\left\{e^{-m x}\right\} f_{+}$is in $L_{1}$. Similarly for $[T] f_{-}$.

We may apply the preceding result to obtain a formula for the Fourier transform of any pansion of exponential growth:

96.5. Theorem. Let $\phi$ be a pansion of exponential growth. Then we may write $\phi=[\cos a D] f$ where $f$ is a function of exponential growth and hence satisfies the conditions of 96.4 for suitable $m$ and $p$. Using the notation introduced above one has

$$
[T]_{\phi}=\left[e^{i m D}\right]\{\cosh a(x-i m)\} T\left\{e^{-m x}\right\} f_{+}+\left[e^{-i p D}\right]\{\cosh a(x+i p)\} T\left\{e^{p x}\right\} f_{-} \text {. }
$$

The proof follows from (62.5), 96.4 and 55.2.

\section{REFERENCES}

1. R. Bellman, Stability theory of differential equations, New York, 1953.

2. R. P. Boas, Jr., Entire functions, New York, 1954.

3. S. Bochner, Vorlesungen über Fouriersche Integrale, Leipzig, 1932.

4. E. A. Coddington and N. Levinson, Theory of ordinary differential equations, New York, 1955.

5. P. A. M. Dirac, The principles of quantum mechanics, Oxford, 1930, 1947.

6. G. Doetsch, Theorie und Anwendung der Laplace Transformation, Berlin, 1937.

7. L. Ehrenpreis, Analytic functions and the Fourier transform of distributions I, Ann. of Math. (2) vol. 63 (1956) pp. 129-159.

8. I. M. Gel'fand and G. E. Silov, Fourier transforms of rapidly increasing functions and questions of uniqueness of the solution of Cauchy's problem, Uspehi Mat. Nauk (N.S.) vol. 8 (58) (1953) pp. 3-54.

9. I. I. Hirschman and D. V. Widder, Convolution transforms, Princeton, 1955.

10. L. Infeld and T. E. Hull, The factorization method, Rev. Mod. Phys. vol. 23 (1951) pp. 21-68.

11. J. Korevaar, Distributions defined by fundamental sequences, Nederl. Akad. Wetensch. Proc. Ser. A vol. 58 (1955) pp. 368-389, 483-503, 663-674.

12. - Fourier transforms of generalized functions, Proceedings of the Symposium on Harmonic Analysis, Cornell University, 1956, Ithaca, 1957, vol. 2, pp. 1-43.

13. R. E. A. C. Paley and N. Wiener, Fourier transforms in the complex domain, New York, 1934.

14. S. Saks, Theory of the integral, New York, 1937.

15. E. Schrödinger, $A$ method of determining quantum-mechanical eigenvalues and eigenfunctions, Proc. Roy. Irish Acad. Sect. A vol. 46 (1940) pp. 9-16.

16. L. Schwartz, Theorie des distributions, I, II, Paris, 1950, 1951.

17. G. Szegö, Orthogonal polynomials, New York, 1939. 
18. E. C. Titchmarsh, The theory of functions, Oxford, 1932, 1939.

19. - Introduction to the theory of Fourier integrals, Oxford, 1937, 1948.

20. H. Weyl, The theory of groups and quantum mechanics, New York, 1931.

21. N. Wiener, The Fourier integral and certain of its applications, Cambridge, 1933.

The University of Wisconsin,

Madison, Wis. 